\title{
Behavior of Tubular Flange Girder System in Curved Overpasses
}

\author{
Asal Mohammed Mahdi ${ }^{1}$, Ammar A. Abdul Rahman ${ }^{2}$ \\ ${ }^{1}$ M.Sc. Graduate, Civil Engineering Department, Al Nahrain University, Baghdad, IRAQ \\ ${ }^{2}$ Ph.D. Structural Engineering, Faculty Member, Civil Engineering Department, Al Nahrain University, Baghdad, IRAQ
}

\begin{abstract}
Curved overpasses with steel girders are now widely used in Iraq because they add significant flexibility in the determination of highway alignments, especially at congested interchanges inside major cities. The horizontal curvature produces significant torsional effects in the bridge girder system. In this study new types of curved steel girders are proposed to make the system stable against this torsional effect where their behavior in curved bridge systems is investigated and compared with the typical I-section girder under the same geometry and loading conditions. These new types of girders have a tube in place of the bottom flange of the I-section girder. Four models are proposed herein. The finite element program SAP2000 version 14 is used for the linear analysis of the horizontally curved simply supported composite bridge decks with the proposed girder models and the reference I-section girder. The factors considered in the parametric study are: span length, curvature, number of mid diaphragms and girder type. The results include the longitudinal stresses and the vertical displacements in the mid span of the bridge. These results have been compared with those of the reference Isection girder. The results showed that the proposed models give less stresses and deflections than the typical I-section and the most effective model in curved deck bridge is girder model $D$ (the bottom plate is made of steel channel with plates inclined with $45^{\circ}$ we Ided together instead of the bottom steel plate and filled with concrete).
\end{abstract}

Keywords: Curved Steel tubular flange Girder, SAP2000, AASHTO.

\section{Introduction}

Traffic jam problems became the most significant concern inside major cities in Iraq; curved overpasses with steel girders are now widely used in Iraq because they add significant flexibility in the determination of highway alignments, especially at congested interchanges inside major cities. The horizontal curvature produces significant torsional effects in the bridge girder system. Four new models of steel girders are proposed. These girders have tubular shapes in place of the bottom flange plate of I-girders and these tubular flanges will be hollow or filled with concrete. The proposed models of tubular flange girders will be studied to investigate their behavior in steel curved bridges for the typical span lengths of overpasses in Iraq. The models behavior will be compared with I- section girders with the same dimensions. The tubular flange girder models and the reference I-section girder are shown in Figure (1).

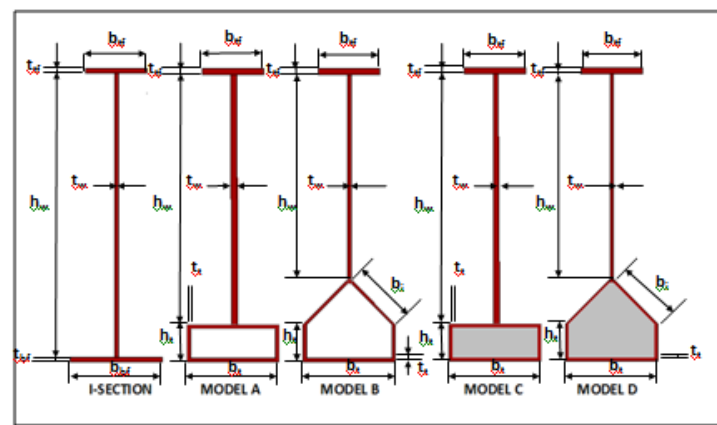

Figure 1: The tubular flange girders types and I-section.

The study is based on the following assumptions

1) Composite Action: the reinforced concrete slab deck will behave in full interaction with the steel girder.

2) The spans under consideration are simply-supported.
3) All materials are linear, elastic and homogenous.

4) Sections selected are designed for section compactness, strength, buckling and local torsional capacities based on AASHTO- ASD, $17^{\text {th }}$ Edition, 2001 [1].

5) The effect of road super-elevation and curbs are neglected.

6) The study does not include the effect of cyclic and fatigue loadings.

7) The effect of friction forces between deck slab and girders is neglected.

Other bridge configurations are listed below:

a)Three spans will be adopted which are the typical spans used in Iraq. These spans are (24, 30 and 36) $\mathrm{m}$.

b) The deck slab thickness $\left(\mathrm{t}_{\mathrm{s}}\right)$ is taken as $(0.22 \mathrm{~m})$.

c) The carriageway width is taken equal to $(7 \mathrm{~m})$ with sidewalks of $(1 \mathrm{~m})$ on each side of the bridge .The total width of the bridge is equal to $(9 \mathrm{~m})$.

d)Number of girders supporting the deck is 4 .

e) The spacing between the girders is $2.2 \mathrm{~m}$.

f) Three lines of headed shear stud connectors with (22 mm) in diameter are designed based on article (10.38.2) of AASHTO-ASD specifications $17^{\text {th }}$ Edition ,2001 [1], so that the behavior of the composite action is full interaction (slip is very small and considered negligible). The spacing between shear studs for span $(24 \mathrm{~m})$ is $(0.4 \mathrm{~m})$ and the spacing for the spans $(30 \mathrm{~m})$ and $(36 \mathrm{~m})$ is $(0.3 \mathrm{~m})$. These spacings are from sites work information of available bridges in Iraq.

g) The girder web thickness is considered equal to $16 \mathrm{~mm}$.

h)Diaphragms are made of K-type truss bracing, the top chord bracing is UPN140 and bottom chord bracings are ) $\mathrm{L} 100 * 100 * 10 \mathrm{~mm}$ ) and the diagonal bracing is ( $\mathrm{L}$ $75 * 75 * 7 \mathrm{~mm})$.

i) The wearing surface density is $\left(22 \mathrm{kN} / \mathrm{m}^{3}\right)$ and the thickness will be assumed (100 mm). 


\section{International Journal of Science and Research (IJSR) \\ ISSN (Online): 2319-7064}

Index Copernicus Value (2013): 6.14 | Impact Factor (2014): 5.611

j) The material properties of the components of the composite bridge deck are specified in Table(1) below:

Table 1: Material properties of composite bridge deck

\begin{tabular}{|c|c|c|}
\multicolumn{3}{|c|}{ Components } \\
\hline$E_{c}$ & Concrete & 24682 \\
\hline$f c^{`}$ & Compressive strength $(\mathrm{MPa})$ & 27.58 \\
\hline$v$ & Poisson's ratio & 0.2 \\
\hline$\gamma_{c}$ & Density of fill concrete $\left(\mathrm{kN} / \mathrm{m}^{3}\right)$ & 24 \\
\hline$\gamma_{c}$ & Density of reinforced concrete deck $\left(\mathrm{kN} / \mathrm{m}^{3}\right)$ & 24.5 \\
\hline & Steel & \\
\hline Type & A 50 steel & - \\
\hline$E_{s}$ & Young's modulus $(\mathrm{MPa})$ & 200000 \\
\hline$f y$ & Yield stress (MPa) & 345 \\
\hline$v$ & Poisson's ratio & 0.3 \\
\hline$\gamma_{s}$ & Density $\left(\mathrm{kN} / \mathrm{m}^{3}\right)$ & 78.6 \\
\hline
\end{tabular}

Figure (2) shows the cross section details of the typical composite bridge deck. K-type cross-bracings with top and bottom chords are utilized in this study. Typical plan of curved girders with the distribution of the radial bracings are shown in Figure (3) for span $24 \mathrm{~m}$.

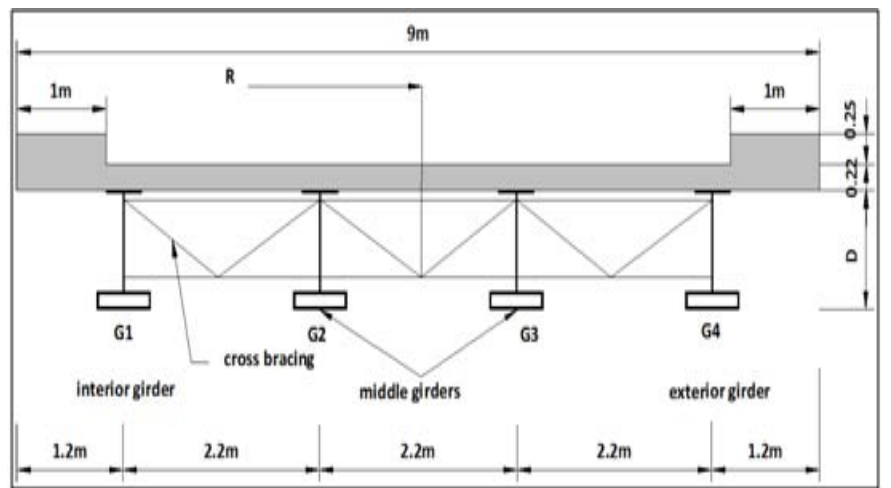

Figure 2: Cross sectional view of composite bridge model with model A steel girders.

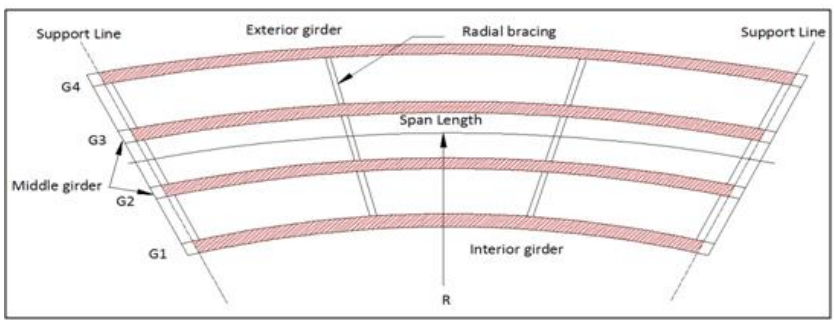

Figure 3: Top plan of curved span of $24 \mathrm{~m}$ showing radial bracings

\section{Bridge Loading}

According to AASHTO LRFD -2004[2], the highway live loadings on the roadways of bridges or incidental structures shall consist of standard trucks and lane loads that are equivalent to truck trains. One type of loading is provided in our study, Truck Loading (HL93), the heaviest truck available in AASHTO LRFD -2004. Bridge configurations considered in this study include two full trucks loading one in each notational lane with the other wheel load located $0.6 \mathrm{~m}$ from the curb at exterior side of deck as considered by AASHTO LRFD specification. Figure (4) shows the transverse location of loading for composite curved bridge.

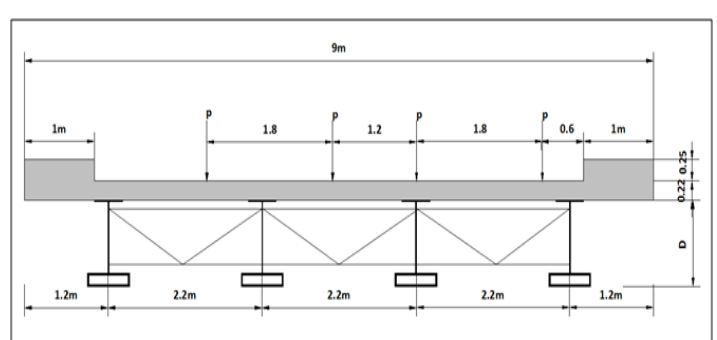

Figure 4: Transverse load location for composite bridge with model A steel girders

\section{Finite Element Analysis}

\subsection{Finite Element Models}

To analyze the composite bridges and to determine their structural behavior, a three-dimensional finite-element model is used. The composite bridge is divided into concrete deck slab, top steel flange, steel web, bottom steel tube, and the cross-bracings. Four-node shell elements with six degrees of freedom at each node are used to model the concrete deck slab, the top flange, bottom tube girder, and finally the web. Frame elements, pinned at both ends, are used to model the cross-bracings with the top and bottom chords. Solid elements are used to model the concrete in the case of tube filled with concrete. Shear stud connectors can be modeled as shell element with same projected area along the top flange of the girder to connect the concrete deck slab to girder top flange .The real composite action where the slab bears slip (even small value) between the concrete deck slab and top steel flange is best simulated in this modeling. Figure (5) shows the three dimensional view of the curved bridge and the coordinate system.

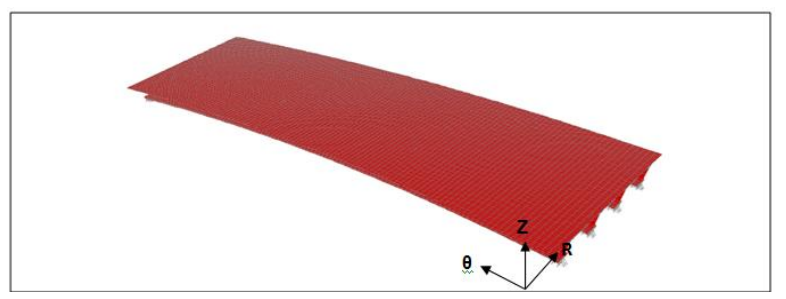

Figure 5: Three dimensional view of the curved bridge and coordinate system.

\subsection{Boundary Conditions}

The bridge supports modeling used in this study is to select the middle lower nodes of the lower face of the bottom flange of the tube in both $\mathrm{x}$ and $\mathrm{y}$ directions fixed to represent the bearing pads which have dimensions of $(200 * 200) \mathrm{mm}$. The interior support at the right end of the bridge is restrained against movements in all directions. The middle supports and the exterior support at the same right end of the bridge are restrained against the vertical movement and against the movement in y-direction (towards the bridge longitudinal direction). For the other end of the bridge (left end), all the supports are only restrained against vertical movement, except for the interior support which in addition to the vertical restraining, it is restrained in $\mathrm{x}$ direction (towards the bridge transverse direction), see Figure (6) . 


\section{International Journal of Science and Research (IJSR) ISSN (Online): 2319-7064}

Index Copernicus Value (2013): 6.14 | Impact Factor (2014): 5.611

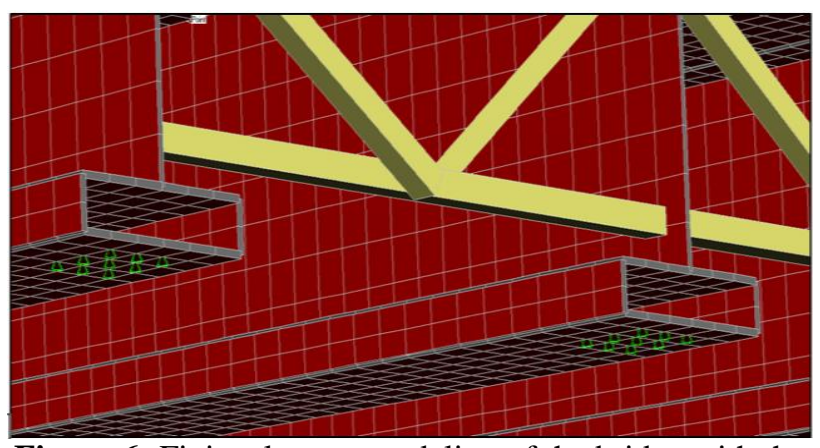

Figure 6: Finite element modeling of the bridge with the boundary conditions

\subsection{Applied Loads}

The applied loads are shown in Table (2) below:

Table 2: Types of loading

\begin{tabular}{|c|c|}
\hline Type of loading & Values \\
\hline Dead load & Self Weight +Weight Of Wearing Coarse \\
\hline Live load & Truck HL 93 Load \\
\hline
\end{tabular}

SAP2000 accepts loading the structures at the nodes with concentrated load or on the shell element as uniform loading. For truck loading HL93it is applied as concentrated loads on nodes at the top of the slab in position to get the maximum effect for flexural stresses at the mid-span location as shown in Figure (7).

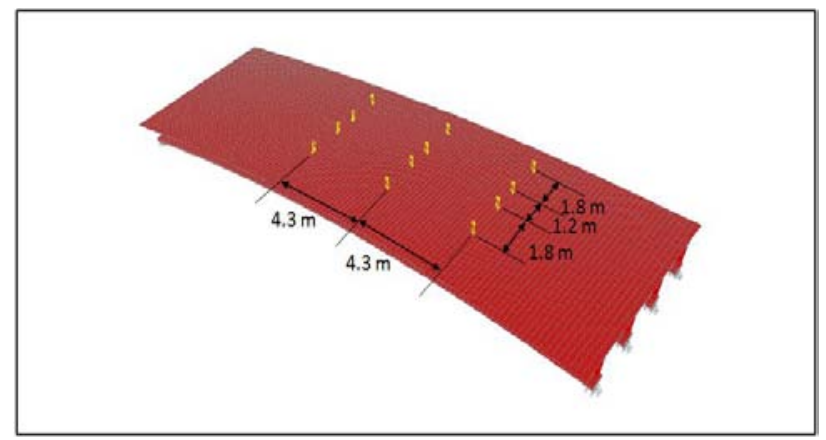

Figure 7: Applied loading of HL93 Truck Load

\section{Parametric Study}

The horizontal curvature produces significant torsional effects in the bridge girder system. For this purpose, researchers and designers to investigate and find new sections having the ability to resist this torsion and to make the system more stable against the applied load. This is the objective of this study; hence, for this purpose the proposed models described previously will be examined and their results will be compared with the normal I-section girder. The important parameters affecting stresses and displacements. The parameters chosen for this study are the span length, span-to-radius ratio, number of bracings and the steel girder shape and these parameters are shown in Table (3) .The width of bridge; number of girders and the spacing between girders are kept constant in this study.
Table 3: Parameters for all proposed four models and Isection girders with (end bracings only)

\begin{tabular}{|c|c|c|c|c|}
\hline $\begin{array}{c}\text { Span } \\
(m)\end{array}$ & $\begin{array}{c}L / R \\
\text { Ratio }\end{array}$ & $\begin{array}{c}R \\
(m)\end{array}$ & $\begin{array}{c}\text { number of } \\
\text { cross bracing } \\
\text { (at ends only) }\end{array}$ & $\begin{array}{c}\text { number of cross } \\
\text { bracing (additional } \\
\text { mid) }\end{array}$ \\
\hline 24 & 0.1 & 240 & 2 & 4 \\
\hline & 0.2 & 120 & 2 & 4 \\
\hline & 0.3 & 80 & 2 & 4 \\
\hline & 0.4 & 60 & 2 & 4 \\
\hline 30 & 0.1 & 300 & 2 & 6 \\
\hline & 0.2 & 150 & 2 & 6 \\
\hline & 0.3 & 100 & 2 & 6 \\
\hline & 0.4 & 75 & 2 & 6 \\
\hline 36 & 0.1 & 360 & 2 & 7 \\
\hline & 0.2 & 180 & 2 & 7 \\
\hline & 0.3 & 120 & 2 & 7 \\
\hline & 0.4 & 90 & 2 & 7 \\
\hline
\end{tabular}

\subsection{Results}

\subsubsection{Stresses}

After analysis of the four proposed models and the typical Isection girder, the stresses are calculated. The longitudinal bending stresses in the bottom flange and concrete deck slab of the proposed models are compared with I-section girder stresses to know the behavior of these steel girders under dead and live load (two trucks of HL93). All these results are measured in the mid span for the interior and exterior girders G1 and G4 respectively. Tables (4) to (27) show the bottom longitudinal stresses in bottom flange and top longitudinal stresses in concrete slab deck for the proposed models and Isection and for the exterior G4 and interior G1 girders and for all parameters. The results show that the stresses in the proposed models are in general less than the stresses of Isection girders under the same loading and same parameters. This difference between the results is mostly because the moment of inertia and the torsional rigidity of the proposed models are greater than those in I-section especially in model $\mathrm{C}$ and model $\mathrm{D}$. The difference in stresses between proposed models and I-section indicate that the proposed models can carry more loads than I-section. The stresses in I-section girder increase in large amount but in proposed models they are increased but with much smaller amounts. Also the results show how the effect of cross bracing on stresses which show that when there are mid cross bracings the stresses are small in all sections and when the mid cross bracing is removed the stresses are increased in large amount in I-section girder but in very small amount in proposed models. This means that mid bracing in bridges with the proposed models can be minimized. Figures (8) to (17) show the longitudinal stresses in the bottom flange and concrete deck slab for span $36 \mathrm{~m}$ and $(\mathrm{L} / \mathrm{R}=0.4)$ which is the critical case in this study for all proposed models including Isections and for all parameters Figures (18) to Figure (23) show the relation between the longitudinal stresses in the bottom flange of I-section and the proposed models with $(\mathrm{L} / \mathrm{R})$ ratio. Notations given in the following table are used:

\begin{tabular}{|c|c|}
\hline Notation & Meaning \\
\hline+ & Tensile Stresses \\
\hline- & Compression Stresses \\
\hline$\sigma_{\mathrm{Bf}}$ & Bending Stresses In Bottom Steel Flange \\
\hline$\sigma_{\mathrm{ts}}$ & Bending Stresses In Top Face Of Concrete Deck Slab \\
\hline
\end{tabular}




\section{International Journal of Science and Research (IJSR) ISSN (Online): 2319-7064 \\ Index Copernicus Value (2013): 6.14 | Impact Factor (2014): 5.611}

Table 4: Longitudinal normal stresses in the bottom flange and concrete deck slab For girders of span $24 \mathrm{~m}\left(\mathrm{~N} / \mathrm{mm}^{2}\right)$ (with 2 mid bracings) with $(\mathrm{L} / \mathrm{R}=0.1)$

\begin{tabular}{|c|c|c|c|c|}
\hline G/R ratio & \multicolumn{2}{|c|}{ G1 } & \multicolumn{2}{|c|}{ G4 } \\
\hline 0.1 & $\sigma_{\text {bf }}$ & $\sigma_{\text {ts }}$ & $\sigma_{\text {bf }}$ & $\sigma_{\text {ts }}$ \\
\hline I-SECTION & 92.048 & -2.22 & 118.692 & -2.964 \\
\hline Model A & 70.852 & -2.652 & 91.786 & -3.519 \\
\hline Model B & 79.226 & -2.888 & 100.824 & -3.649 \\
\hline Model C & 46.56 & -2.038 & 58.765 & -2.464 \\
\hline Model D & 46.098 & -1.944 & 55.857 & -2.363 \\
\hline
\end{tabular}

Table 5: Longitudinal normal stresses in the bottom flange and concrete deck slab for girders of span $24 \mathrm{~m}\left(\mathrm{~N} / \mathrm{mm}^{2}\right)$ (with 2 mid bracings) with $(\mathrm{L} / \mathrm{R}=0.2)$

\begin{tabular}{|c|c|c|c|c|}
\hline Girder & \multicolumn{2}{|c|}{ G1 } & \multicolumn{2}{c|}{ G4 } \\
\hline L/R ratio & $\boldsymbol{\sigma}_{\text {bf }}$ & $\boldsymbol{\sigma}_{\text {ts }}$ & $\boldsymbol{\sigma}_{\text {bf }}$ & $\boldsymbol{\sigma}_{\text {ts }}$ \\
\hline I-SECTION & 118.46 & -2.607 & 174.919 & -3.98 \\
\hline Model A & 69.453 & -2.579 & 114.521 & -3.761 \\
\hline Model B & 77.067 & -2.919 & 110.947 & -4.147 \\
\hline Model C & 55.196 & -2.299 & 78.391 & -3.155 \\
\hline Model D & 57.845 & -2.499 & 76.469 & -3.181 \\
\hline
\end{tabular}

Table 6: Longitudinal normal stresses in the bottom flange and concrete deck slab for girders of span $24 \mathrm{~m}\left(\mathrm{~N} / \mathrm{mm}^{2}\right)$ (with 2 mid bracings) with $(\mathrm{L} / \mathrm{R}=0.3)$

\begin{tabular}{|c|c|c|c|c|}
\hline Girder & \multicolumn{2}{|c|}{ G1 } & \multicolumn{2}{c|}{ G4 } \\
\hline L/R ratio & $\boldsymbol{0}_{\text {bf }}$ & $\boldsymbol{\sigma}_{\text {ts }}$ & $\boldsymbol{\sigma}_{\text {bf }}$ & $\boldsymbol{\sigma}_{\text {ts }}$ \\
\hline I-SECTION & 123.23 & -2.455 & 205.529 & -4.344 \\
\hline Model A & 67.848 & -2.474 & 116.058 & -4.1 \\
\hline Model B & 74.19 & -2.929 & 118.756 & -4.462 \\
\hline Model C & 55.395 & -2.143 & 86.547 & -3.299 \\
\hline Model D & 57.883 & -2.368 & 81.965 & -3.225 \\
\hline
\end{tabular}

Table 7: Longitudinal normal stresses in the bottom flange and concrete deck slab for girders of span $24 \mathrm{~m}\left(\mathrm{~N} / \mathrm{mm}^{2}\right)$ (with 2 mid bracings) with ( $\mathrm{L} / \mathrm{R}=0.4)$

\begin{tabular}{|c|c|c|c|c|}
\hline Girder & \multicolumn{2}{|c|}{ G1 } & \multicolumn{2}{c|}{ G4 } \\
L/R ratio & $\boldsymbol{\sigma}_{\text {bf }}$ & $\boldsymbol{\sigma}_{\text {ts }}$ & $\boldsymbol{\sigma}_{\text {bf }}$ & $\boldsymbol{\sigma}_{\text {ts }}$ \\
\hline I-SECTION & 122.567 & -2.308 & 228.469 & -4.461 \\
\hline Model A & 64.899 & -2.345 & 124.766 & -4.362 \\
\hline Model B & 76.572 & -3.012 & 131.742 & -4.821 \\
\hline Model C & 64.638 & -2.103 & 110.335 & -3.632 \\
\hline Model D & 61.719 & -2.237 & 91.657 & -3.195 \\
\hline
\end{tabular}

Table 8: longitudinal normal stresses in the bottom flange and concrete deck slab for girders of span $24 \mathrm{~m}\left(\mathrm{~N} / \mathrm{mm}^{2}\right)$ (with end bracings only) with $(\mathrm{L} / \mathrm{R}=0.1)$

\begin{tabular}{|c|c|c|c|c|}
\hline Girder & \multicolumn{2}{|c|}{ G1 } & \multicolumn{2}{c|}{ G4 } \\
\hline L/R ratio & $\boldsymbol{\sigma}_{\text {bf }}$ & $\boldsymbol{\sigma}_{\text {ts }}$ & $\boldsymbol{\sigma}_{\text {bf }}$ & $\boldsymbol{\sigma}_{\text {ts }}$ \\
\hline I-SECTION & 109.376 & -2.501 & 131.83 & -2.984 \\
\hline Model A & 77.016 & -2.948 & 94.302 & -3.44 \\
\hline Model B & 80.913 & -2.966 & 99.505 & -3.607 \\
\hline Model C & 49.485 & -2.099 & 59.731 & -2.391 \\
\hline Model D & 45.979 & -1.957 & 54.66 & -2.323 \\
\hline
\end{tabular}

Table 9: Longitudinal normal stresses in the bottom flange and concrete deck slab for girders of span $24 \mathrm{~m}\left(\mathrm{~N} / \mathrm{mm}^{2}\right)$ (with end bracings only) with $(\mathrm{L} / \mathrm{R}=0.2)$

\begin{tabular}{|c|c|c|c|c|}
\hline L/R ratio & \multicolumn{2}{|c|}{ G1 } & \multicolumn{2}{|c|}{ G4 } \\
\hline 0.2 & $\sigma_{\text {bf }}$ & $\sigma_{\mathrm{ts}}$ & $\sigma_{\text {bf }}$ & $\sigma_{\text {ts }}$ \\
\hline I-SECTION & 166.95 & -3.736 & 202.297 & -4.61 \\
\hline Model A & 84.357 & -3.45 & 108.881 & -3.984 \\
\hline Model B & 81.767 & -3.262 & 108.033 & -4.324 \\
\hline Model C & 64.356 & -2.71 & 81.082 & -3.147 \\
\hline Model D & 60.367 & -2.706 & 75.988 & -3.225 \\
\hline
\end{tabular}

Table 10: Longitudinal normal stresses in the bottom flange and concrete deck slab for girders of span $24 \mathrm{~m}\left(\mathrm{~N} / \mathrm{mm}^{2}\right)$

(with end bracings only) with $(\mathrm{L} / \mathrm{R}=0.3)$

\begin{tabular}{|c|c|c|c|c|}
\hline L/R ratio & \multicolumn{2}{|c|}{ G1 } & \multicolumn{2}{|c|}{ G4 } \\
\hline 0.3 & $\sigma_{\text {bf }}$ & $\sigma_{\text {ts }}$ & $\sigma_{\text {bf }}$ & $\sigma_{\mathrm{ts}}$ \\
\hline I-SECTION & 193.539 & -4.338 & 234.952 & -5.504 \\
\hline Model A & 92.319 & -4.048 & 121.21 & -4.709 \\
\hline Model B & 82.764 & -3.848 & 114.445 & -4.667 \\
\hline Model C & 69.909 & -2.99 & 89.579 & -3.44 \\
\hline Model D & 62.265 & -2.716 & 81.271 & -3.189 \\
\hline
\end{tabular}

Table 11: Longitudinal normal stresses in the bottom flange and concrete deck slab for girders of span $24 \mathrm{~m}\left(\mathrm{~N} / \mathrm{mm}^{2}\right)$ (with end bracings only) with $(\mathrm{L} / \mathrm{R}=0.4)$

\begin{tabular}{|c|c|c|c|c|}
\hline Girder & \multicolumn{2}{|c|}{ G1 } & \multicolumn{2}{c|}{ G4 } \\
\hline L/R ratio & $\boldsymbol{\sigma}_{\text {bf }}$ & $\boldsymbol{\sigma}_{\text {ts }}$ & $\boldsymbol{\sigma}_{\text {bf }}$ & $\boldsymbol{\sigma}_{\text {ts }}$ \\
\hline I-SECTION & 211.913 & -4.975 & 239.306 & -6.294 \\
\hline Model A & 118.34 & -4.709 & 152.352 & -5.299 \\
\hline Model B & 91.903 & -4.342 & 129.515 & -5.29 \\
\hline Model C & 95.003 & -3.538 & 120.944 & -3.932 \\
\hline Model D & 69.274 & -2.687 & 91.21 & -3.179 \\
\hline
\end{tabular}

Table 12: Longitudinal normal stresses in the bottom flange and concrete deck slab for girders of span $30 \mathrm{~m}\left(\mathrm{~N} / \mathrm{mm}^{2}\right)$ (with 4 mid bracings) with (L/R=0.1)

\begin{tabular}{|c|c|c|c|c|}
\hline${ }_{\text {L/R ratio }}^{\text {Girder }}$ & \multicolumn{2}{|c|}{ G1 } & \multicolumn{2}{|c|}{ G4 } \\
\hline 0.1 & $\sigma_{\text {bf }}$ & $\sigma_{\mathrm{ts}}$ & $\sigma_{\text {bf }}$ & $\sigma_{\text {ts }}$ \\
\hline I-SECTION & 97.545 & -2.62 & 131.272 & -3.689 \\
\hline Model A & 73.46 & -2.919 & 99.921 & -4.013 \\
\hline Model B & 80.289 & -3.115 & 106.992 & -4.153 \\
\hline Model C & 53.342 & -2.405 & 69.738 & -3.207 \\
\hline Model D & 52.013 & -2.488 & 65.364 & -3.146 \\
\hline
\end{tabular}

Table 13: Longitudinal normal stresses in the bottom flange and concrete deck slab for girders of span $30 \mathrm{~m}\left(\mathrm{~N} / \mathrm{mm}^{2}\right)$ (with 4 mid bracings) with $(\mathrm{L} / \mathrm{R}=0.2)$

\begin{tabular}{|c|r|c|c|c|}
\hline Girder & \multicolumn{2}{|c|}{ G1 } & \multicolumn{2}{c|}{ G4 } \\
\hline L/R ratio & $\boldsymbol{\sigma}_{\text {bf }}$ & $\boldsymbol{\sigma}_{\text {ts }}$ & $\boldsymbol{\sigma}_{\text {bf }}$ & $\boldsymbol{\sigma}_{\text {ts }}$ \\
\hline I-SECTION & 97.286 & -2.569 & 158.529 & -4.552 \\
\hline Model A & 69.05 & -2.51 & 116.459 & -4.512 \\
\hline Model B & 74.289 & -2.877 & 112.493 & -4.675 \\
\hline Model C & 55.757 & -2.428 & 86.562 & -4.018 \\
\hline Model D & 57.755 & -2.766 & 83.037 & -4.167 \\
\hline
\end{tabular}




\section{International Journal of Science and Research (IJSR) \\ ISSN (Online): 2319-7064 \\ Index Copernicus Value (2013): 6.14 | Impact Factor (2014): 5.611}

Table 14: Longitudinal normal stresses in the bottom flange and concrete deck slab for girders of span $30 \mathrm{~m}\left(\mathrm{~N} / \mathrm{mm}^{2}\right)$ (with 4 mid bracings) with $(\mathrm{L} / \mathrm{R}=0.3)$

\begin{tabular}{|c|c|c|c|c|}
\hline Girder & \multicolumn{2}{|c|}{ G1 } & \multicolumn{2}{c|}{ G4 } \\
\hline L/R ratio & $\boldsymbol{\sigma}_{\text {bf }}$ & $\boldsymbol{\sigma}_{\text {ts }}$ & $\boldsymbol{\sigma}_{\text {bf }}$ & $\boldsymbol{\sigma}_{\text {ts }}$ \\
\hline 0.3 & 77.094 & -1.783 & 154.549 & -4.406 \\
\hline I-SECTION & 54.008 & -1.806 & 112.706 & -4.191 \\
\hline Model A & 59.247 & -1.928 & 117.945 & -4.276 \\
\hline Model B & 59.785 & -1.807 & 89.812 & -3.77 \\
\hline Model C & 48.78 & -1.972 & 88.743 & -3.501 \\
\hline Model D & 47.716 & \multicolumn{3}{|c}{} \\
\hline
\end{tabular}

Table (15): Longitudinal normal stresses in the bottom flange and concrete deck slab for girders of span $30 \mathrm{~m}$ $\left(\mathrm{N} / \mathrm{mm}^{2}\right)$ (with 4 mid bracings) with $(\mathrm{L} / \mathrm{R}=0.4)$

\begin{tabular}{|c|c|c|c|c|}
\hline Girder & \multicolumn{2}{|c|}{ G1 } & \multicolumn{2}{c|}{ G4 } \\
\hline L/R ratio & $\boldsymbol{\sigma}_{\text {bf }}$ & $\boldsymbol{\sigma}_{\text {ts }}$ & \multicolumn{1}{c|}{$\boldsymbol{\sigma}_{\text {bf }}$} & $\boldsymbol{\sigma}_{\text {ts }}$ \\
\hline 0.4 & 94.747 & -1.803 & 215.671 & -4.89 \\
\hline I-SECTION & 92.696 & -1.951 & 143.698 & -4.902 \\
\hline Model A & 62.151 & -2.402 & 142.034 & -5.189 \\
\hline Model B & 67.151 & -3.718 \\
\hline Model C & 53.003 & -1.702 & 88.2 & -3.495 \\
\hline Model D & 50.875 & -2.032 & 91.604 & - \\
\hline
\end{tabular}

Table 16: Longitudinal normal stresses in the bottom flange and concrete deck slab for girders of span $30 \mathrm{~m}\left(\mathrm{~N} / \mathrm{mm}^{2}\right)$ (with end bracings only) with $(\mathrm{L} / \mathrm{R}=0.1)$

\begin{tabular}{|c|l|l|l|c|}
\hline Girder & \multicolumn{2}{|c|}{ G1 } & \multicolumn{2}{c|}{ G4 } \\
\hline L/R ratio & \multicolumn{1}{|c|}{$\boldsymbol{\sigma}_{\text {bf }}$} & $\boldsymbol{\sigma}_{\text {ts }}$ & $\boldsymbol{\sigma}_{\text {bf }}$ & $\boldsymbol{\sigma}_{\text {ts }}$ \\
\hline I-SECTION & 124.654 & -3.028 & 166.244 & -4.559 \\
\hline Model A & 91.241 & -3.405 & 110.084 & -3.803 \\
\hline Model B & 89.935 & -3.443 & 108.937 & -3.957 \\
\hline Model C & 61.9 & -2.706 & 77.763 & -3.006 \\
\hline Model D & 56.041 & -2.616 & 68.176 & -2.998 \\
\hline
\end{tabular}

Table 17: Longitudinal normal stresses in the bottom flange and concrete deck slab for girders of span $30 \mathrm{~m}\left(\mathrm{~N} / \mathrm{mm}^{2}\right)$ (with end bracings only) with ( $\mathrm{L} / \mathrm{R}=0.2)$

\begin{tabular}{|c|l|c|c|c|}
\hline Girder & \multicolumn{2}{|c|}{ G1 } & \multicolumn{2}{c|}{ G4 } \\
\hline L/R ratio & $\boldsymbol{\sigma}_{\text {bf }}$ & $\boldsymbol{\sigma}_{\text {ts }}$ & $\boldsymbol{\sigma}_{\text {bf }}$ & $\boldsymbol{\sigma}_{\text {ts }}$ \\
\hline $\mathbf{0 . 2}$ & 185.146 & -4.439 & 222.719 & -5.643 \\
\hline I-SECTION & 108.323 & -3.958 & 137.104 & -4.878 \\
\hline Model A & 13.587 & -3.836 & 126.327 & -4.793 \\
\hline Model B & 91.58 & -3.422 & 102.863 & -3.849 \\
\hline Model C & 84.546 & -3.257 & 88.976 & -4.118 \\
\hline Model D & 72.6354 & - &
\end{tabular}

Table 18: Longitudinal normal stresses in the bottom flange and concrete deck slab for girders of span $30 \mathrm{~m}\left(\mathrm{~N} / \mathrm{mm}^{2}\right)$ (with end bracings only) with $(\mathrm{L} / \mathrm{R}=0.3)$

\begin{tabular}{|c|c|c|c|c|}
\hline Girder & \multicolumn{2}{|c|}{ G1 } & \multicolumn{2}{c|}{ G4 } \\
\hline L/R ratio & $\boldsymbol{\sigma}_{\text {bf }}$ & $\boldsymbol{\sigma}_{\text {ts }}$ & $\boldsymbol{\sigma}_{\text {bf }}$ & $\boldsymbol{\sigma}_{\text {ts }}$ \\
\hline 0.3 & 187.38 & -4.503 & 224.261 & -6.732 \\
\hline I-SECTION & 105.194 & -3.812 & 138.426 & -5.282 \\
\hline Model A & 100.476 & -3.934 & 133.708 & -5.367 \\
\hline Model B & 100.416 & -3.177 & 112.199 & -4.149 \\
\hline Model C & 78.9128 & -3.342 & 105.429 & -3.88 \\
\hline Model D & 72.1428 & -4 & \multicolumn{1}{|c}{}
\end{tabular}

Table 19: Longitudinal normal stresses in the bottom flange and concrete deck slab for girders of span $30 \mathrm{~m}\left(\mathrm{~N} / \mathrm{mm}^{2}\right)$ (with end bracings only) with $(\mathrm{L} / \mathrm{R}=0.4)$

\begin{tabular}{|c|c|c|c|c|}
\hline Girder & \multicolumn{2}{|c|}{ G1 } & \multicolumn{2}{c|}{ G4 } \\
\hline L/R ratio & $\boldsymbol{\sigma}_{\text {bf }}$ & $\boldsymbol{\sigma}_{\text {ts }}$ & $\boldsymbol{\sigma}_{\text {bf }}$ & $\boldsymbol{\sigma}_{\text {ts }}$ \\
\hline 0.4 & 200.017 & -6.039 & 270.597 & -7.505 \\
\hline I-SECTION & 126.005 & -5.522 & 179.281 & -6.358 \\
\hline Model A & 126.51 & -5.993 \\
\hline Model B & 114.237 & -4.931 & 153.712 & -3.799 \\
\hline Model C & 102.847 & -3.563 & 121.59 & -3.79 \\
\hline Model D & 80.727 & -2.911 & 101.385 & -3.367 \\
\hline
\end{tabular}

Table 20: Longitudinal normal stresses in the bottom flange and concrete deck slab for girders of span $36 \mathrm{~m}\left(\mathrm{~N} / \mathrm{mm}^{2}\right)$ (with 5 mid bracings) with $(\mathrm{L} / \mathrm{R}=0.1)$

\begin{tabular}{|c|c|c|c|c|}
\hline Girder & \multicolumn{2}{|c|}{ G1 } & \multicolumn{2}{c|}{ G4 } \\
\hline L/R ratio & $\boldsymbol{\sigma}_{\text {bf }}$ & $\boldsymbol{\sigma}_{\text {ts }}$ & $\boldsymbol{\sigma}_{\text {bf }}$ & $\boldsymbol{\sigma}_{\text {ts }}$ \\
\hline 0.1 & 93.826 & -2.534 & 127.695 & -3.546 \\
\hline MoCTION & 77.149 & -3.122 & 108.119 & -4.429 \\
\hline Model A & 85.493 & -3.315 & 117.024 & -4.509 \\
\hline Model C & 53.839 & -2.397 & 71.743 & -3.192 \\
\hline Model D & 50.482 & -2.394 & 64.417 & -3.039 \\
\hline
\end{tabular}

Table 21: Longitudinal normal stresses in the bottom flange and concrete deck slab for girders of span $36 \mathrm{~m}\left(\mathrm{~N} / \mathrm{mm}^{2}\right)$ (with 5 mid bracings) with $(\mathrm{L} / \mathrm{R}=0.2)$

\begin{tabular}{|c|c|c|c|c|}
\hline $\begin{array}{c}\text { Girder } \\
\text { L/R ratio }\end{array}$ & \multicolumn{2}{|c|}{ G1 } & \multicolumn{2}{|c|}{ G4 } \\
\hline 0.2 & $\sigma_{\text {bf }}$ & $\sigma_{\text {ts }}$ & $\sigma_{\text {bf }}$ & $\sigma_{\text {ts }}$ \\
\hline I-SECTION & 86.433 & -3.025 & 140.077 & -3.649 \\
\hline Model A & 72.647 & -2.621 & 126.227 & -4.826 \\
\hline Model B & 78.242 & -2.878 & 130.583 & -4.98 \\
\hline Model C & 50.171 & -2.115 & 78.192 & -3.322 \\
\hline Model D & 48.207 & -2.68 & 69.417 & -3.086 \\
\hline
\end{tabular}

Table 22: Longitudinal normal stresses in the bottom flange and concrete deck slab for girders of span $36 \mathrm{~m}\left(\mathrm{~N} / \mathrm{mm}^{2}\right)$ (with 5 mid bracings) with ( $\mathrm{L} / \mathrm{R}=0.3$ )

\begin{tabular}{|c|c|c|c|c|}
\hline $\begin{array}{c}\text { Girder } \\
L / R \text { ratio }\end{array}$ & \multicolumn{2}{|c|}{ G1 } & \multicolumn{2}{|c|}{ G4 } \\
\hline 0.3 & $\sigma_{\text {bf }}$ & $\sigma_{\mathrm{ts}}$ & $\sigma_{\text {bf }}$ & $\sigma_{\mathrm{ts}}$ \\
\hline I-SECTION & 96.989 & -1.984 & 209.378 & -6.705 \\
\hline Model A & 51.618 & -2.146 & 107.174 & -7.133 \\
\hline Model B & 67.456 & -2.637 & 143.073 & -5.66 \\
\hline Model C & 53.793 & -2.066 & 104.119 & -5.046 \\
\hline Model D & 54.805 & -2.508 & 95.249 & -4.82 \\
\hline
\end{tabular}

Table 23: Longitudinal normal stresses in the bottom flange and concrete deck slab for girders of span $36 \mathrm{~m}\left(\mathrm{~N} / \mathrm{mm}^{2}\right)$ (with 5 mid bracings) with $(\mathrm{L} / \mathrm{R}=0.4)$

\begin{tabular}{|c|c|c|c|c|}
\hline$\underbrace{\text { Girder }}_{\text {L/R ratio }}$ & \multicolumn{2}{|c|}{ G1 } & \multicolumn{2}{|c|}{ G4 } \\
\hline 0.4 & $\sigma_{\text {bf }}$ & $\sigma_{\text {ts }}$ & $\sigma_{\text {bf }}$ & $\sigma_{\text {ts }}$ \\
\hline I-SECTION & 85.536 & -1.493 & 234.082 & -5.944 \\
\hline Model A & 55.643 & -2.472 & 153.907 & -5.961 \\
\hline Model B & 61.663 & -2.179 & 149.48 & -6.245 \\
\hline Model C & 52.067 & -1.695 & 117.466 & -5.156 \\
\hline Model D & 53.562 & -2.620 & 100.498 & -4.627 \\
\hline
\end{tabular}




\section{International Journal of Science and Research (IJSR) \\ ISSN (Online): 2319-7064 \\ Index Copernicus Value (2013): 6.14 | Impact Factor (2014): 5.611}

Table 24: Longitudinal normal stresses in the bottom flange and concrete deck slab for girders of span $36 \mathrm{~m}\left(\mathrm{~N} / \mathrm{mm}^{2}\right)$ (with end bracings only) with ( $\mathrm{L} / \mathrm{R}=0.1)$

\begin{tabular}{|c|c|c|c|c|}
\hline Girder & \multicolumn{2}{|c|}{ G1 } & \multicolumn{2}{c|}{ G4 } \\
\hline L/R ratio & $\boldsymbol{\sigma}_{\text {bf }}$ & $\boldsymbol{\sigma}_{\text {ts }}$ & $\boldsymbol{\sigma}_{\text {bf }}$ & $\boldsymbol{\sigma}_{\text {ts }}$ \\
\hline 0.1 & 139.024 & -2.974 & 166.262 & -3.416 \\
\hline I-SECTION & 105.364 & -3.775 & 126.115 & -4.145 \\
\hline Model A & 105.145 & -4.296 \\
\hline Model B & 101.881 & -3.784 & 122.43 & -2.91 \\
\hline Model C & 70.017 & -2.779 & 82.631 & -617 \\
\hline Model D & 59.153 & -2.591 & 69.357 & -2.799 \\
\hline
\end{tabular}

Table 25: Longitudinal normal stresses in the bottom flange and concrete deck slab for girders of span $36 \mathrm{~m}\left(\mathrm{~N} / \mathrm{mm}^{2}\right)$ (with end bracings only) with $(\mathrm{L} / \mathrm{R}=0.2)$

\begin{tabular}{|c|c|c|c|c|}
\hline Girder & \multicolumn{2}{|c|}{ G1 } & \multicolumn{2}{c|}{ G4 } \\
\hline L/R ratio & $\boldsymbol{\sigma}_{\text {bf }}$ & $\boldsymbol{\sigma}_{\text {ts }}$ & $\boldsymbol{\sigma}_{\text {bf }}$ & $\boldsymbol{\sigma}_{\text {ts }}$ \\
\hline I-2 & 119.563 & -2.096 & 154.889 & -3.64 \\
\hline Model A & 127.785 & -4.211 & 156.464 & -4.977 \\
\hline Model B & 113.461 & -3.989 & 142.744 & -4.771 \\
\hline Model C & 72.358 & -2.774 & 95.403 & -2.887 \\
\hline Model D & 65.665 & -2.509 & 78.937 & -2.702 \\
\hline
\end{tabular}

Table 26: Longitudinal normal stresses in the bottom flange and concrete deck slab for girders of span $36 \mathrm{~m}\left(\mathrm{~N} / \mathrm{mm}^{2}\right)$ (with end bracings only) with $(\mathrm{L} / \mathrm{R}=0.3)$

\begin{tabular}{|c|c|c|c|c|}
\hline$\underbrace{\text { Girder }}_{L / R \text { ratio }}$ & \multicolumn{2}{|c|}{ G1 } & \multicolumn{2}{|c|}{ G4 } \\
\hline 0.3 & $\sigma_{\text {bf }}$ & $\sigma_{\text {ts }}$ & $\sigma_{\text {bf }}$ & $\sigma_{\text {ts }}$ \\
\hline I-SECTION & 186.054 & -5.828 & 284.386 & -7.227 \\
\hline Model A & 151.792 & -5.557 & 191.084 & -6.485 \\
\hline Model B & 123.012 & -4.786 & 166.356 & -6.167 \\
\hline Model C & 114.185 & -4.128 & 142.947 & -4.423 \\
\hline Model D & 91.682 & -3.667 & 115.16 & -3.978 \\
\hline
\end{tabular}

Table 27: Longitudinal normal stresses in the bottom flange and concrete deck slab for girders of span $36 \mathrm{~m}\left(\mathrm{~N} / \mathrm{mm}^{2}\right)$ (with end bracings only) with $(\mathrm{L} / \mathrm{R}=0.4)$

\begin{tabular}{|c|c|c|c|c|}
\hline Girder & \multicolumn{2}{|c|}{ G1 } & \multicolumn{2}{c|}{ G4 } \\
\hline L/R ratio & $\boldsymbol{\sigma}_{\text {bf }}$ & $\boldsymbol{\sigma}_{\text {ts }}$ & $\boldsymbol{\sigma}_{\text {bf }}$ & $\boldsymbol{\sigma}_{\text {ts }}$ \\
\hline I-4 & 269.547 & -7.183 & 325.323 & -8.805 \\
\hline Model A & 159.643 & -6.511 & 207.895 & -7.436 \\
\hline Model B & 128.321 & -5.373 & 177.494 & -6.799 \\
\hline Model C & 127.75 & -4.687 & 158.938 & -4.892 \\
\hline Model D & 94.318 & -3.663 & 120.012 & -3.909 \\
\hline
\end{tabular}

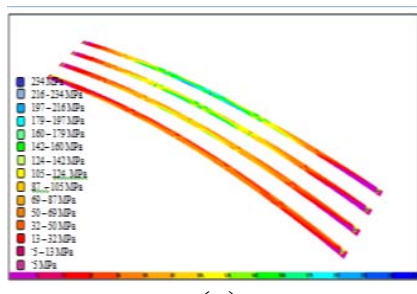

(a)

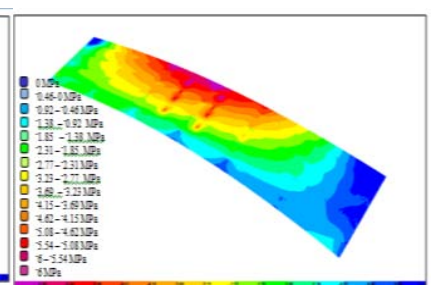

(b)
Figure (8): Longitudinal normal stresses $(\sigma)$ for deck of Isection girders with 5 mid bracings for span $36 \mathrm{~m}(\mathrm{~L} / \mathrm{R}=0.4)$ in the (a) Bottom flange (b) Deck slab.

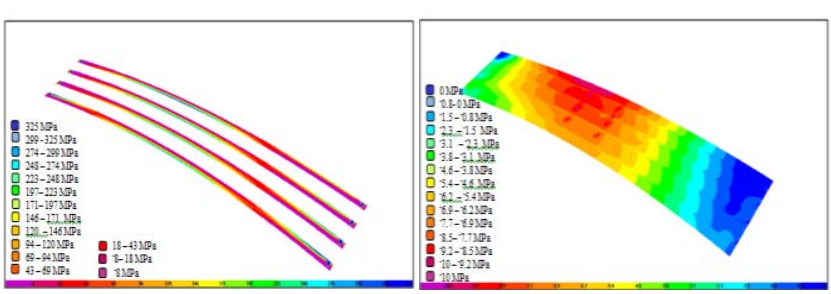

(a)

(b)

Figure 9: Longitudinal normal stresses $(\sigma)$ for deck of Isection girders with end bracings for span $36 \mathrm{~m}(\mathrm{~L} / \mathrm{R}=0.4)$ in the (a) Bottom flange (b) Deck slab

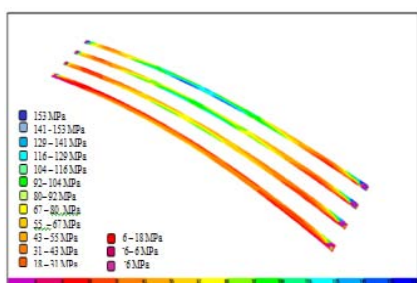

(a)

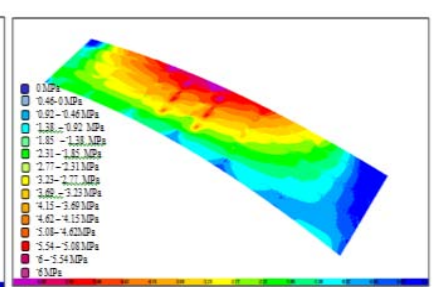

(b)
Figure 10: Longitudinal normal stresses $(\sigma)$ for deck of model A girders with $5 \mathrm{mid}$ bracings for span $36 \mathrm{~m}(\mathrm{~L} / \mathrm{R}=0.4)$ in the (a) Bottom flange (b) Deck slab

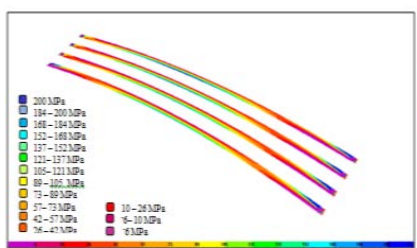

(a)

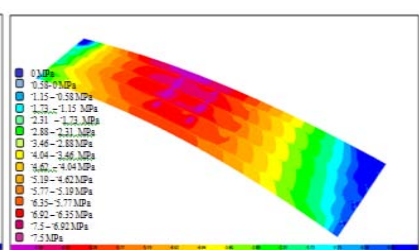

(b)
Figure 11: Longitudinal normal stresses $(\sigma)$ for deck of model A girders with end bracings for span $36 \mathrm{~m}(\mathrm{~L} / \mathrm{R}=0.4)$ in the (a) Bottom flange (b) Deck slab

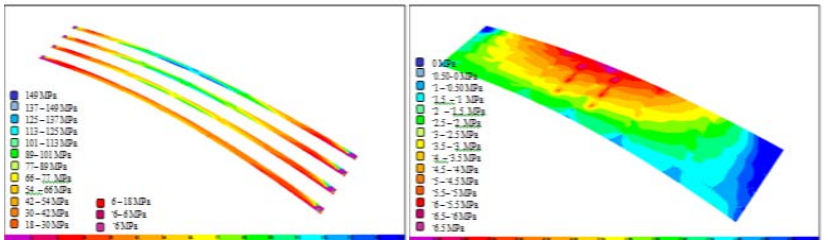

(a)

(b)

Figure 12: Longitudinal normal stresses $(\sigma)$ for deck of model $B$ girders with 5 mid bracings for span $36 \mathrm{~m}(\mathrm{~L} / \mathrm{R}=0.4)$ in the (a) Bottom flange (b) Deck slab

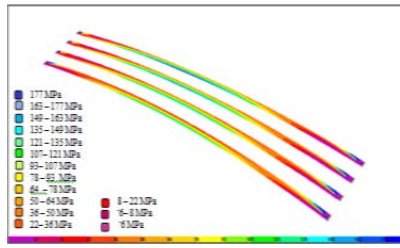

(a)

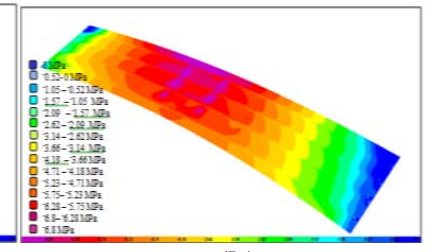

(b)
Figure 13: Longitudinal normal stresses $(\sigma)$ for deck of model $B$ girders with end bracings for span $36 \mathrm{~m}(\mathrm{~L} / \mathrm{R}=0.4)$ in the (a) Bottom flange (b) Deck slab. 


\section{International Journal of Science and Research (IJSR) \\ ISSN (Online): 2319-7064}

Index Copernicus Value (2013): 6.14 | Impact Factor (2014): 5.611

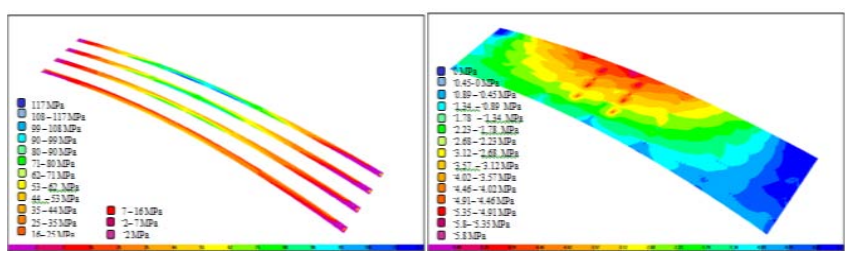

(a)

(b)

Figure 14: Longitudinal normal stresses $(\sigma)$ for deck of model $C$ girders with 5 mid bracings for span $36 \mathrm{~m}(\mathrm{~L} / \mathrm{R}=0.4)$ in the (a) Bottom flange (b) Deck slab

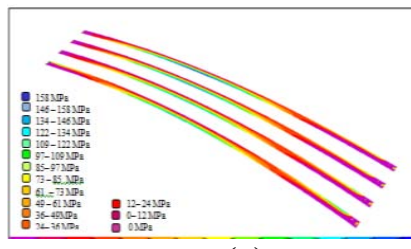

(a)

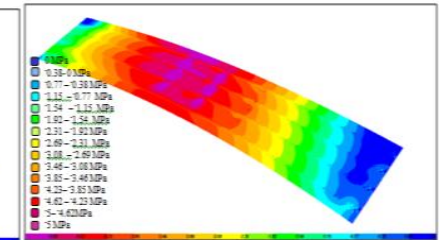

(b)
Figure 15: Longitudinal normal stresses $(\sigma)$ for deck of model $C$ girders with end bracings for span $36 \mathrm{~m}(\mathrm{~L} / \mathrm{R}=0.4)$ in the (a) Bottom flange (b) Deck slab.

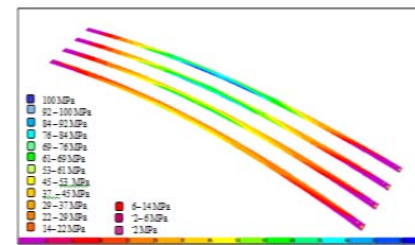

(a)

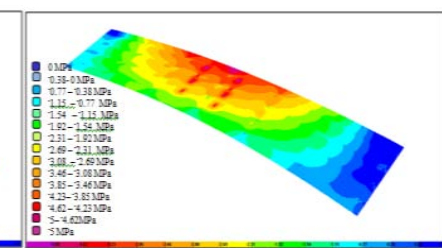

(b)
Figure 16: Longitudinal normal stresses $(\sigma)$ for deck of model D girders with 5 mid bracings for span $36 \mathrm{~m}(\mathrm{~L} / \mathrm{R}=0.4)$ in the (a) Bottom flange (b) Deck slab.

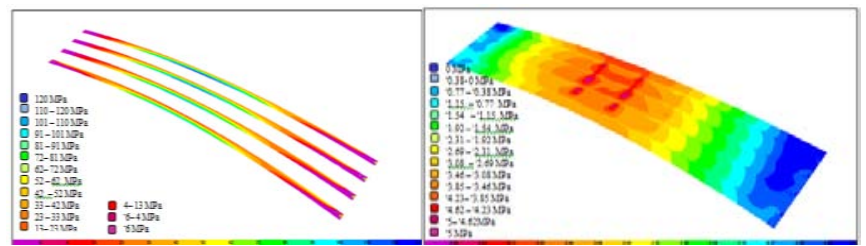

(a)

(b)

Figure 17: Longitudinal normal stresses $(\sigma)$ for deck of model $D$ with end bracings for span $36 \mathrm{~m}(\mathrm{~L} / \mathrm{R}=0.4)$ in the (a) Bottom flange (b) Deck slab.

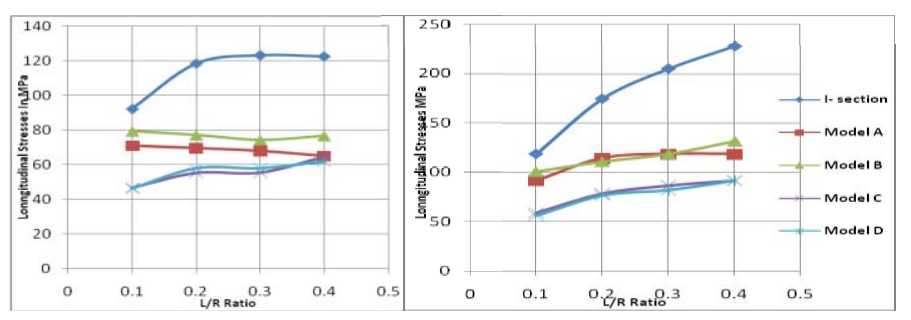

(a)

(b)

Figure 18: Longitudinal normal stresses $(\sigma)$ versus $L / R$ ratio for all proposed models and I-section with $2 \mathrm{mid}$ bracings for span 24m (a) Interior girder (G1) (b) Exterior girder (G4)

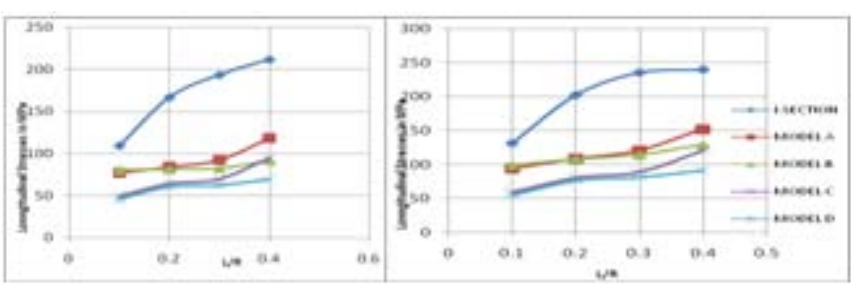

(a)

(b)

Figure 19: Longitudinal normal stresses $(\sigma)$ versus $L / R$ ratio for all proposed models and I-section with end bracings only for span 24m (a) Interior girder (G1) (b) Exterior girder (G4)

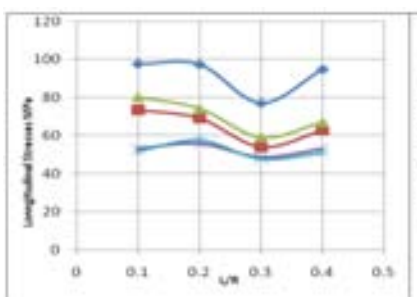

(a)

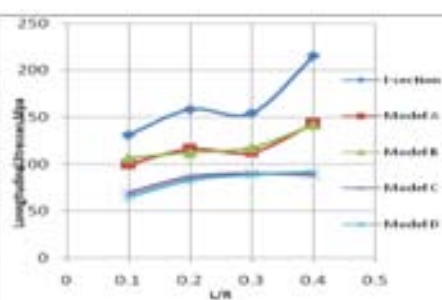

(b)
Figure 20: Longitudinal normal stresses $(\sigma)$ versus $L / R$ ratio for all proposed models and I-section with 4 mid bracings for span 30m (a) Interior girder (G1) (b) Exterior girder (G4).

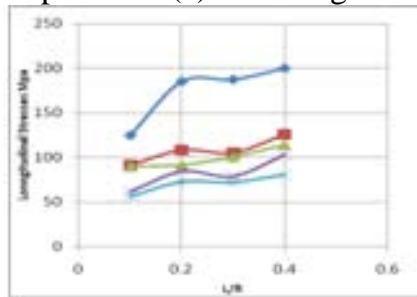

(a)

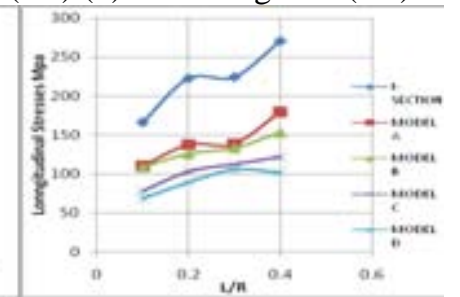

(b)
Figure 21: Longitudinal normal stresses $(\sigma)-\mathrm{L} / \mathrm{R}$ ratio for all proposed models and I-section with end bracings only for span 30m (a) Interior girder (G1) (b) Exterior girder (G4).

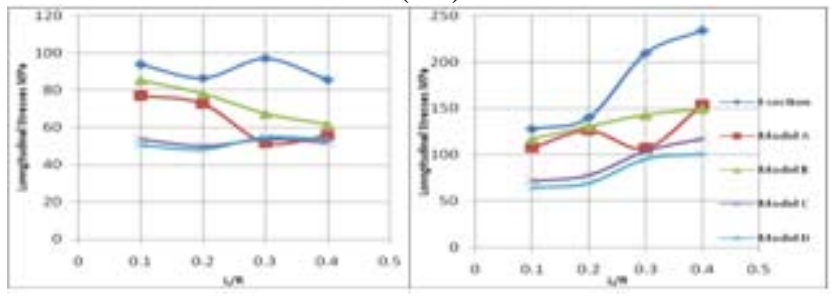

(a)

(b)

Figure 22: Longitudinal normal stresses $(\sigma)$ versus L/R ratio for all proposed models and I-section with 5 mid bracings for span 36m (a) Interior girder (G1) (b) Exterior girder (G4).

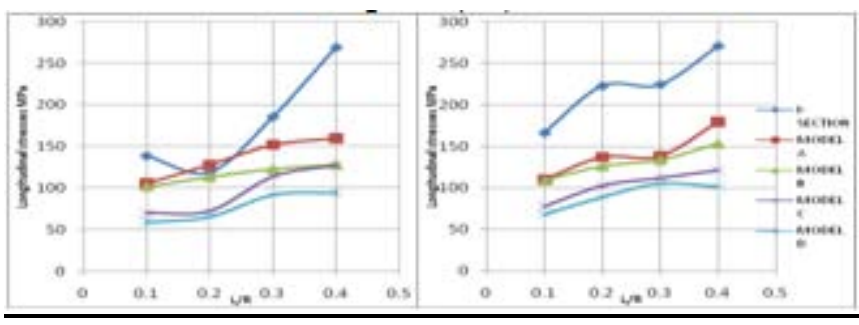

(a)

(b)

Figure 23: Longitudinal normal stresses $(\sigma)$ versus $L / R$ ratio for all proposed models and I-section with end bracings only for span 36m (a) Interior girder (G1) (b) Exterior girder (G4). 


\section{International Journal of Science and Research (IJSR) \\ ISSN (Online): 2319-7064}

Index Copernicus Value (2013): 6.14 | Impact Factor (2014): 5.611

\subsubsection{Vertical Displacements}

From the parameters above the vertical displacements will be found in the vertical direction at the bottom of the girder. All these results are measured in the mid span for the interior and exterior girders. Tables (28) to (33) show the vertical displacement for all parameters. The results show that the vertical displacement for I-section, model A and model B show large displacement than model $\mathrm{C}$ and $\mathrm{D}$ because of the presence of concrete in the tube gives the stability and stiffness to the girder and thus to the whole system. Figure (24) and (25) show the distribution of vertical displacements in the bottom of I-section and proposed model D for the span length (36m) with $(\mathrm{L} / \mathrm{R}=0.4)$. Figures (26) to Figure (31) show the vertical displacements versus $L / R$ ratio in the bottom flange of I-section and the proposed models.

Table 28: Vertical displacement (mm) in mid span of girders for span $24 \mathrm{~m}$ (with 2 mid bracings)

\begin{tabular}{|c|c|c|c|c|c|}
\hline \multicolumn{3}{|c|}{ (L/R=0.1) } & \multicolumn{3}{c|}{ (L/R=0.2) } \\
\hline Section & \multicolumn{2}{|c|}{ Girder } & Section & \multicolumn{2}{c|}{ Girder } \\
\cline { 2 - 3 } Type & G1 & G4 & Type & G1 & G4 \\
\hline I-section & -33.465 & -46.272 & I-section & -41.426 & -70.15 \\
\hline Model A & -44.833 & -63.001 & Model A & -45.662 & -78.17 \\
\hline Model B & -48.226 & -65.440 & Model B & -53.112 & -83.88 \\
\hline Model C & -26.120 & -35.443 & Model C & -31.092 & -50.88 \\
\hline Model D & -24.906 & -32.310 & Model D & -33.047 & -49.60 \\
\hline \multicolumn{3}{|c|}{ (L/R=0.3) } & \multicolumn{3}{|c|}{ (L/R=0.4) } \\
\hline Section & \multicolumn{2}{|c|}{ Girder } & Section & \multicolumn{2}{|c|}{ Girder } \\
\cline { 2 - 3 } Type & G1 & G4 & Type & G1 & G4 \\
\hline I-section & -41.637 & -81.816 & I-section & -41.403 & -91.26 \\
\hline Model A & -46.625 & -93.333 & Model A & -47.078 & -106.4 \\
\hline Model B & -57.059 & -102.93 & Model B & -61.037 & -120.5 \\
\hline Model C & -30.612 & -58.043 & Model C & -32.434 & -70.88 \\
\hline Model D & -32.389 & -54.810 & Model D & -31.324 & -58.60 \\
\hline
\end{tabular}

Table 29: Vertical displacement (mm) in mid span of girders for span $24 \mathrm{~m}$ (with end bracings)

\begin{tabular}{|c|c|c|c|c|c|}
\hline \multicolumn{3}{|c|}{$(\mathrm{L} / \mathrm{R}=\mathbf{0 . 1})$} & \multicolumn{3}{|c|}{$(\mathrm{L} / \mathrm{R}=0.2)$} \\
\hline \multirow{2}{*}{$\begin{array}{c}\text { Section } \\
\text { Type }\end{array}$} & \multicolumn{2}{|c|}{ Girder } & \multirow{2}{*}{$\begin{array}{c}\text { Section } \\
\text { Type }\end{array}$} & \multicolumn{2}{|c|}{ Girder } \\
\hline & G1 & G4 & & G1 & G4 \\
\hline I-section & -41.609 & $\begin{array}{l}-56.527 \\
\end{array}$ & I-section & -69.790 & -107.77 \\
\hline Model A & -49.329 & -65.627 & Model A & -59.651 & -90.694 \\
\hline Model B & -50.636 & -66.376 & Model B & -68.709 & -79.263 \\
\hline Model C & -27.457 & -35.766 & $\mathbf{M}$ & -36.896 & -54.519 \\
\hline Model D & -25.234 & -32.148 & Model D & -34.934 & -50.043 \\
\hline \multicolumn{3}{|c|}{$(\mathrm{L} / \mathrm{R}=0.3)$} & \multicolumn{3}{|c|}{$(\mathrm{L} / \mathrm{R}=0.4)$} \\
\hline \multirow{2}{*}{$\begin{array}{c}\text { Section } \\
\text { Type } \\
\end{array}$} & \multicolumn{2}{|c|}{ Girder } & Section & \multicolumn{2}{|c|}{ Girder } \\
\hline & G1 & G4 & Type & G1 & G1 \\
\hline I-section & -87.649 & -142.96 & I-section & -101.53 & -170.34 \\
\hline Model A & -72.130 & -119.53 & Model A & -84.201 & -147.87 \\
\hline Model B & -71.888 & -115.97 & Model B & -82.912 & -141.48 \\
\hline Model C & -40.785 & -66.206 & Model C & -50.319 & -87.808 \\
\hline Model D & -35.713 & -56.282 & Model D & -36.054 & -61.434 \\
\hline
\end{tabular}

Table 30: Vertical displacement (mm) in mid span of girders for span 30m (with 4 mid bracings)

\begin{tabular}{|c|c|c|c|c|c|}
\hline \multicolumn{3}{|c|}{$(\mathrm{L} / \mathrm{R}=0.1)$} & \multicolumn{3}{|c|}{$(\mathrm{L} / \mathrm{R}=0.2)$} \\
\hline \multirow{2}{*}{$\begin{array}{c}\text { Section } \\
\text { Type }\end{array}$} & \multicolumn{2}{|c|}{ Girder } & \multirow{2}{*}{$\begin{array}{l}\text { Section } \\
\text { Type }\end{array}$} & \multicolumn{2}{|c|}{ Girder } \\
\hline & G1 & G4 & & G1 & G4 \\
\hline I-section & -44.409 & -62.705 & I-section & -47.62 & -84.315 \\
\hline Model A & -52.305 & -75.047 & Model A & -52.146 & -95.271 \\
\hline Model B & -55.553 & -77.293 & Model B & -58.25 & -97.33 \\
\hline Model C & -34.350 & -47.915 & Model C & -37.427 & -66.293 \\
\hline Model D & -34.279 & -45.566 & Model D & -41.011 & -67.187 \\
\hline \multicolumn{3}{|c|}{$(\mathrm{L} / \mathrm{R}=\mathbf{0 . 3})$} & \multicolumn{3}{|c|}{$(\mathrm{L} / \mathrm{R}=0.4)$} \\
\hline
\end{tabular}

\begin{tabular}{|l|c|c|l|c|c|}
\hline \multirow{2}{*}{$\begin{array}{c}\text { Section } \\
\text { Type }\end{array}$} & \multicolumn{2}{|c|}{ Girder } & \multirow{2}{*}{ ection Type } & \multicolumn{2}{c|}{ Girder } \\
\cline { 2 - 5 } & G1 & G4 & & G1 & G4 \\
\hline I-section & -40.693 & -88.815 & I-section & -45.387 & -109.96 \\
\hline Model A & -43.329 & -98.279 & Model A & -50.008 & -126.95 \\
\hline Model B & -45.321 & -133.69 & Model B & -61.973 & -139.12 \\
\hline Model C & -31.711 & -68.872 & Model C & -33.088 & -77.295 \\
\hline Model D & -30.123 & -67.421 & Model D & -34.644 & -70.327 \\
\hline
\end{tabular}

Table 31: Vertical displacement (mm) in mid span of girders for span 30m (with end bracings)

\begin{tabular}{|c|c|c|c|c|c|}
\hline \multicolumn{3}{|c|}{$(\mathrm{L} / \mathrm{R}=\mathbf{0 . 1})$} & \multicolumn{3}{|c|}{$(\mathrm{L} / \mathrm{R}=0.2)$} \\
\hline \multirow{2}{*}{$\begin{array}{c}\text { Section } \\
\text { Type }\end{array}$} & \multicolumn{2}{|c|}{ Girder } & \multirow{2}{*}{$\begin{array}{c}\text { Section } \\
\text { Type }\end{array}$} & \multicolumn{2}{|c|}{ Girder } \\
\hline & G1 & G4 & & G1 & G4 \\
\hline I-section & -63.004 & 5.662 & I-section & -103.42 & 154.1 \\
\hline Mod & & & & 34 & 12 \\
\hline Model B & -61 & & B & -79 . & -117.21 \\
\hline Mod & -38. & & & -53.694 & -78.356 \\
\hline $\mathbf{M}$ & -36.085 & -4 & & -50.131 & -71.396 \\
\hline \multicolumn{3}{|c|}{$(\mathrm{L} / \mathrm{R}=\mathbf{0 . 3})$} & \multicolumn{3}{|c|}{$(\mathrm{L} / \mathrm{R}=0.4)$} \\
\hline \multirow{2}{*}{$\begin{array}{c}\text { Section } \\
\text { Type }\end{array}$} & \multicolumn{2}{|c|}{ Girder } & ion & \multicolumn{2}{|c|}{ Girder } \\
\hline & G1 & G4 & & G1 & G1 \\
\hline I-section & -129.49 & -202.84 & I-section & -166.11 & -261.54 \\
\hline & -92.197 & -150.14 & A & -128.71 & -212.86 \\
\hline & -86.240 & -149.22 & & -118.41 & -192.72 \\
\hline & & & & -64.881 & -104.7 \\
\hline Model D & -56.348 & -89.541 & Model D & -50.035 & -80.09 \\
\hline
\end{tabular}

Table 32: Vertical displacement (mm) in mid span of girders for span 36m (with 5 mid bracings)

\begin{tabular}{|c|c|c|c|c|c|}
\hline \multicolumn{3}{|c|}{ (L/R=0.1) } & \multicolumn{3}{c|}{$(\mathbf{L} / \mathbf{R}=\mathbf{0 . 2})$} \\
\hline Section & \multicolumn{2}{|c|}{ Girder } & Section & \multicolumn{2}{c|}{ Girder } \\
\cline { 2 - 3 } Type & G1 & G4 & Type & G1 & G4 \\
\hline I-section & -51.871 & -72.722 & I-section & -45.94 & -78.13 \\
\hline Model A & -64.875 & -95.102 & Model A & -59.041 & -111.63 \\
\hline Model B & -68.681 & -97.492 & Model B & -64.833 & -115.10 \\
\hline Model C & -41.208 & -57.572 & Model C & -37.313 & -63.684 \\
\hline Model D & -39.331 & -52.340 & Model D & -36.162 & -56.672 \\
\hline \multicolumn{3}{|c|}{ (L/R=0.3) } & \multicolumn{3}{|c|}{$(\mathbf{L} / \mathbf{R}=\mathbf{0 . 4})$} \\
\hline Section & \multicolumn{2}{|c|}{ Girder } & Section & \multicolumn{2}{c|}{ Girder } \\
\cline { 2 - 3 } Type & G1 & G4 & Type & G1 & G1 \\
\hline I-section & -61.235 & -135.50 & I-section & -58.706 & -153.98 \\
\hline Model A & -63.308 & -148.82 & Model A & -60.477 & -169.91 \\
\hline Model B & -72.726 & -156.91 & Model B & -71.508 & -177.44 \\
\hline Model C & -45.165 & -99.374 & Model C & -43.685 & -113.01 \\
\hline Model D & -47.030 & -92.240 & Model D & -64.430 & -97.458 \\
\hline
\end{tabular}

Table 33: Vertical displacement (mm) in mid span of girders for span $36 \mathrm{~m}$ (with end bracings)

\begin{tabular}{|c|c|c|c|c|c|}
\hline \multicolumn{3}{|c|}{$(\mathrm{L} / \mathrm{R}=\mathbf{0 . 1})$} & \multicolumn{3}{|c|}{$(\mathrm{L} / \mathrm{R}=0.2)$} \\
\hline \multirow{2}{*}{$\begin{array}{c}\text { Section } \\
\text { Type }\end{array}$} & \multicolumn{2}{|c|}{ Girder } & \multirow{2}{*}{$\begin{array}{c}\text { Section } \\
\text { Type }\end{array}$} & \multicolumn{2}{|c|}{ Girder } \\
\hline & G1 & G4 & & G1 & G4 \\
\hline I-section & -76.465 & -101.37 & I-section & -95.234 & $\begin{array}{l}-131.83 \\
\end{array}$ \\
\hline Model A & -80.437 & -105.71 & Model A & -102.31 & -151.20 \\
\hline Model B & -79.398 & -102.42 & Model B & -94.873 & -137.20 \\
\hline Model C & -47.806 & -60.774 & Model C & -53.849 & -74.768 \\
\hline Model D & -42.339 & -52.574 & Model D & -44.139 & -59.805 \\
\hline \multicolumn{3}{|c|}{$(\mathbf{L} / \mathbf{R}=\mathbf{0 . 3})$} & \multicolumn{3}{|c|}{$(\mathrm{L} / \mathrm{R}=0.4)$} \\
\hline \multirow{2}{*}{$\begin{array}{c}\text { Section } \\
\text { Type }\end{array}$} & \multicolumn{2}{|c|}{ Girder } & Section & \multicolumn{2}{|c|}{ Girder } \\
\hline & G1 & G4 & Type & G1 & G1 \\
\hline I-section & -202.69 & -298.14 & I-section & -262.80 & -395.92 \\
\hline Model A & -158.53 & -247.98 & Model A & -197.34 & -315.55 \\
\hline Model B & -142.99 & -221.24 & Model B & -175.31 & -278.59 \\
\hline Model C & -92.578 & -139.85 & Model C & -106.14 & -181.69 \\
\hline Model D & -72.845 & -108.67 & Model D & -77.4 & -122.57 \\
\hline
\end{tabular}




\section{International Journal of Science and Research (IJSR) ISSN (Online): 2319-7064}

Index Copernicus Value (2013): 6.14 | Impact Factor (2014): 5.611

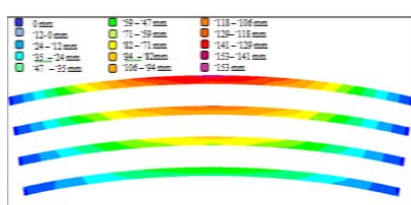

(a)

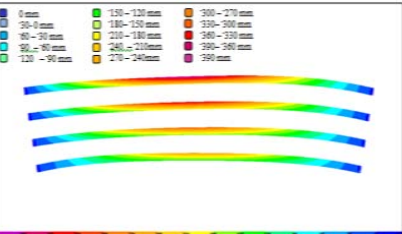

(b)
Figure 24: distribution of vertical displacements in the bottom flange of I-section for the span length (36m) $(\mathrm{L} / \mathrm{R}=0.4$ ) with (a) $5 \mathrm{mid}$ bracings (b) End bracings. (a)

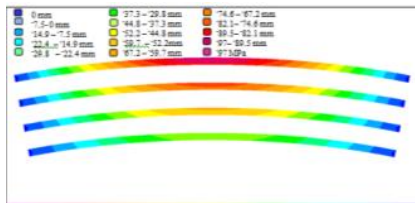

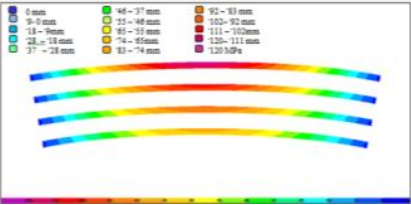

(b)
Figure 25: Distribution of vertical displacements in the bottom flange of model $\mathrm{D}$ for the span length $(36 \mathrm{~m})$ $(\mathrm{L} / \mathrm{R}=0.4$ ) with (a) $5 \mathrm{mid}$ bracings (b) End bracings.

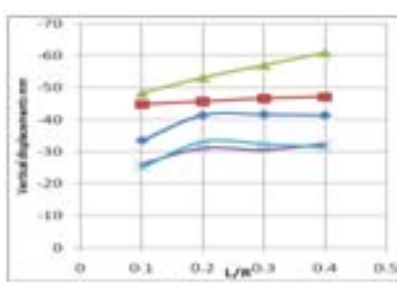

(a)

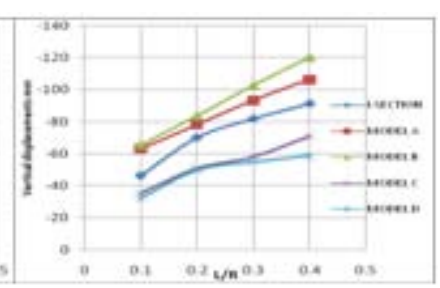

(b)
Figure (26): Vertical displacements versus L/R ratio for all proposed models and I-section with 2 mid bracings for span 24m (a) Interior girder (G1) (b) Exterior girder (G4).

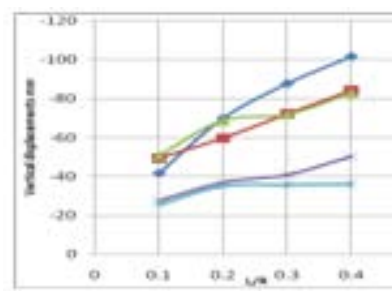

(a)

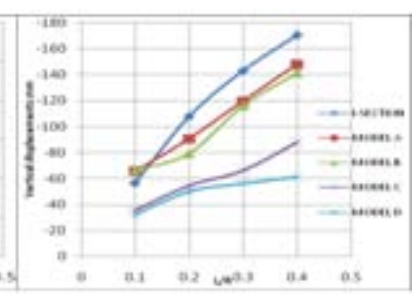

(b)
Figure 27: Vertical displacements versus L/R ratio for all proposed models and I-section with end bracings only for span 24m (a) Interior girder (G1) (b) Exterior girder (G4).

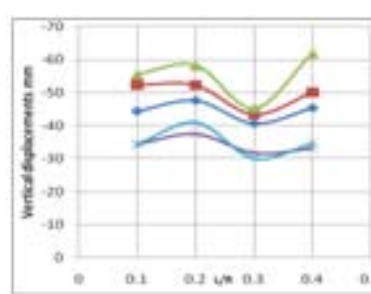

(a)

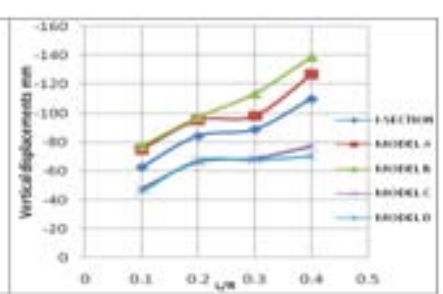

(b)
Figure 28: Vertical displacements versus L/R ratio for all proposed models and I-section with 4 mid bracings for span 30m (a) Interior girder (G1) (b) Exterior girder (G4).

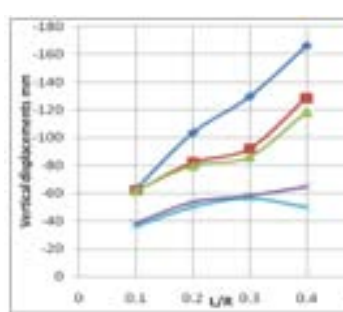

(a)

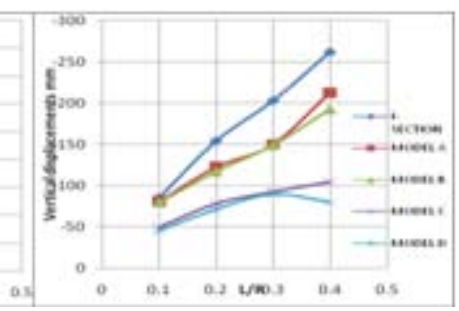

(b)
Figure 29: Vertical displacements versus L/R ratio for all proposed models and I-section with end bracings only for span 30m (a) Interior girder (G1) (b) Exterior girder (G4).

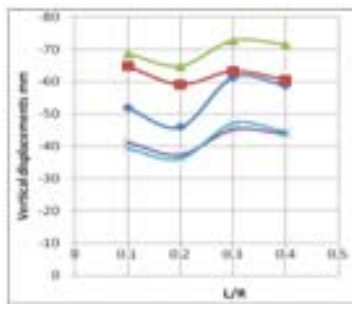

(a)

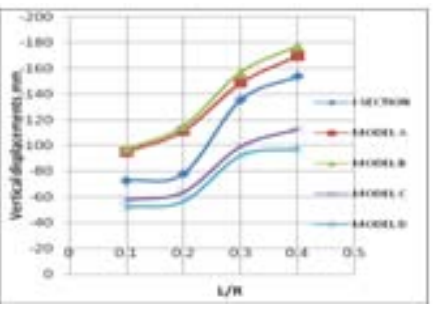

(b)
Figure 30: Vertical displacements versus $L / R$ ratio for all proposed models and I-section with 5 mid bracings for span 36m (a) Interior girder (G1) (b) Exterior girder (G4).

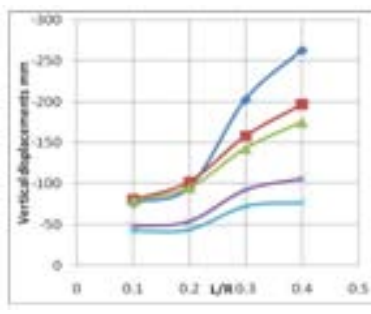

(a)

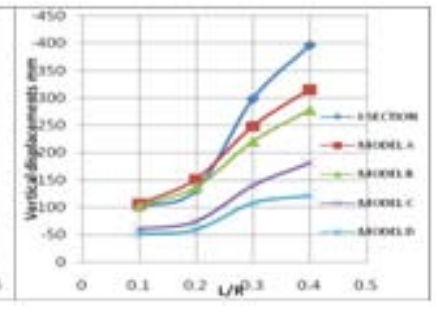

(b)
Figure 31: Vertical displacements versus $\mathrm{L} / \mathrm{R}$ ratio for all proposed models and I-section with end bracings only for span 36m (a) Interior girder (G1) (b) Exterior girder (G4).

\subsection{Comparative Study}

Tables (34) to (36) show the ratio of longitudinal tensile stresses in the bottom flange and compressive stresses in concrete deck slab for the I-section and proposed models between decks modeled with different number of cross bracing. These results are measured at mid span.

Table 34: Span $24 \mathrm{~m}$

\begin{tabular}{|c|c|c|c|c|c|}
\hline \multicolumn{6}{|c|}{$(\mathrm{L} / \mathrm{R}=\mathbf{0 . 1})$} \\
\hline $\begin{array}{c}\text { Ratio Of }\left(\sigma_{\mathrm{Bf}}\right) \\
\text { With(Additional } \\
\text { Mid / End) } \\
\text { Bracings }\end{array}$ & $\begin{array}{c}\text { G1 } \\
\%\end{array}$ & $\begin{array}{l}\text { G4 } \\
\%\end{array}$ & $\begin{array}{l}\text { Ratio Of }\left(\sigma_{\mathrm{ts}}\right) \\
\text { (Additional } \\
\text { Mid / End) } \\
\text { Bracings }\end{array}$ & $\begin{array}{c}\text { G1 } \\
\text { \% }\end{array}$ & $\begin{array}{c}\text { G4 } \\
\%\end{array}$ \\
\hline I-section & 84.16 & 90.03 & I-section & 88.8 & 99.3 \\
\hline Model A & 92.00 & 97.33 & Model A & 90.0 & 102.3 \\
\hline Model B & 97.92 & 101.3 & Model B & 97.1 & 103.1 \\
\hline Model C & 94.09 & 98.38 & Model C & 97.4 & 101.2 \\
\hline Model D & 100.2 & 102.1 & Model D & 99.3 & 101.7 \\
\hline \multicolumn{6}{|c|}{$(\mathrm{L} / \mathrm{R}=0.2)$} \\
\hline $\begin{array}{c}\text { Ratio Of }\left(\sigma_{\mathrm{Bf}}\right) \\
\text { With(Additional } \\
\text { Mid / End) } \\
\text { Bracings }\end{array}$ & $\begin{array}{c}\text { G1 } \\
\%\end{array}$ & $\begin{array}{l}\text { G4 } \\
\%\end{array}$ & $\begin{array}{l}\text { Ratio Of }\left(\sigma_{\mathrm{ts}}\right) \\
\text { (Additional } \\
\text { Mid / End) } \\
\text { Bracings }\end{array}$ & $\begin{array}{c}\text { G1 } \\
\text { \% }\end{array}$ & $\begin{array}{c}\text { G4 } \\
\%\end{array}$ \\
\hline I-section & 70.95 & 86.47 & I-section & 69.8 & 86.3 \\
\hline Model A & 82.33 & 105.2 & Model A & 74.8 & 94.4 \\
\hline Model B & 94.25 & 102.7 & Model B & 84.8 & 100.1 \\
\hline
\end{tabular}


International Journal of Science and Research (IJSR)

ISSN (Online): 2319-7064

Index Copernicus Value (2013): 6.14 | Impact Factor (2014): 5.611

\begin{tabular}{|c|c|c|c|c|c|}
\hline Model C & 85.77 & 96.68 & Model C & 89.5 & 95.9 \\
\hline Model D & 95.82 & 100.6 & Model D & 92.4 & 98.6 \\
\hline \multicolumn{6}{|c|}{$(\mathrm{L} / \mathrm{R}=0.3)$} \\
\hline $\begin{array}{c}\text { Ratio Of }\left(\sigma_{\mathrm{Bf}}\right) \\
\text { With(Additional } \\
\text { Mid / End) } \\
\text { Bracings }\end{array}$ & $\begin{array}{c}\text { G1 } \\
\%\end{array}$ & $\begin{array}{c}\text { G4 } \\
\%\end{array}$ & \begin{tabular}{|} 
Ratio Of $\left(\sigma_{\text {ts }}\right.$ \\
(Additional Mid \\
/ End) Bracings
\end{tabular} & $\begin{array}{c}\text { G1 } \\
\%\end{array}$ & $\begin{array}{c}\text { G4 } \\
\%\end{array}$ \\
\hline I-section & 63.67 & 87.48 & I-section & 56.6 & 78.9 \\
\hline Model A & 73.49 & 95.75 & Model A & 61.1 & 87.1 \\
\hline Model B & 89.64 & 103.7 & Model B & 71.7 & 95.6 \\
\hline Model C & 79.24 & 96.62 & Model C & 76.0 & 95.6 \\
\hline Model D & 92.96 & 100.8 & Model D & \begin{tabular}{|l|}
87.2 \\
\end{tabular} & 101.1 \\
\hline \multicolumn{6}{|c|}{$(\mathrm{L} / \mathrm{R}=\mathbf{0 . 4})$} \\
\hline $\begin{array}{c}\text { Ratio Of }\left(\sigma_{\mathrm{Bf}}\right) \\
\text { With(Additional } \\
\text { Mid / End) } \\
\text { Bracings } \\
\end{array}$ & $\begin{array}{c}\text { G1 } \\
\%\end{array}$ & $\begin{array}{c}\text { G4 } \\
\%\end{array}$ & \begin{tabular}{|c|} 
Ratio Of $\left(\sigma_{\text {ts }}\right.$ \\
(Additional Mid \\
/ End) Bracings
\end{tabular} & $\begin{array}{c}\text { G1 } \\
\%\end{array}$ & $\begin{array}{c}\text { G4 } \\
\%\end{array}$ \\
\hline I-section & 57.84 & 95.47 & I-section & 46.4 & 70.9 \\
\hline Model A & 54.82 & 81.89 & Model A & \begin{tabular}{|l|}
49.8 \\
\end{tabular} & 82.3 \\
\hline Model B & 83.32 & 101.7 & Model B & \begin{tabular}{|l|}
59.4 \\
\end{tabular} & 92.4 \\
\hline Model C & 68.04 & 91.23 & Model C & 69.4 & 91.1 \\
\hline Model D & 89.09 & 100.5 & Model D & \begin{tabular}{|l|}
83.3 \\
\end{tabular} & 100.5 \\
\hline
\end{tabular}

Table 35: Span 30m

\begin{tabular}{|c|c|c|c|c|c|}
\hline \multicolumn{6}{|c|}{$(\mathrm{L} / \mathrm{R}=0.1)$} \\
\hline $\begin{array}{c}\text { Ratio Of }\left(\sigma_{\mathrm{Bf}}\right) \\
\text { With(Additional } \\
\text { Mid / End) } \\
\text { Bracings }\end{array}$ & $\begin{array}{l}\text { G1 } \\
\%\end{array}$ & $\begin{array}{c}\text { G4 } \\
\%\end{array}$ & $\begin{array}{c}\text { Ratio Of ( } \sigma \\
\text { ts) } \\
\text { (Additional } \\
\text { Mid / End) } \\
\text { Bracings } \\
\end{array}$ & $\begin{array}{c}\text { G1 } \\
\%\end{array}$ & $\begin{array}{c}\text { G4 } \\
\%\end{array}$ \\
\hline I-section & 78.25 & 78.96 & I-section & 86.5 & 80.9 \\
\hline Model A & 80.51 & 90.77 & Model A & 85.7 & 105.5 \\
\hline Model B & 89.27 & 98.21 & Model B & 90.5 & 105.0 \\
\hline Model C & 86.17 & 89.68 & Model C & 89.0 & 106.7 \\
\hline Model D & 92.81 & 95.88 & Model D & 94.8 & 104.9 \\
\hline \multicolumn{6}{|c|}{$(\mathrm{L} / \mathrm{R}=0.2)$} \\
\hline $\begin{array}{c}\text { Ratio Of }\left(\sigma_{\mathrm{Bf}}\right) \\
\text { With(Additional } \\
\text { Mid / End) } \\
\text { Bracings }\end{array}$ & $\begin{array}{l}\text { G1 } \\
\%\end{array}$ & $\begin{array}{c}\text { G4 } \\
\%\end{array}$ & $\begin{array}{c}\text { Ratio Of ( } \sigma \\
\text { ts) } \\
\text { (Additional } \\
\text { Mid / End) } \\
\text { Bracings }\end{array}$ & $\begin{array}{c}\text { G1 } \\
\%\end{array}$ & $\begin{array}{c}\text { G4 } \\
\%\end{array}$ \\
\hline I-section & 52.55 & 71.18 & I-section & 57.9 & 80.7 \\
\hline Model A & 63.74 & 84.94 & Model A & 63.4 & 92.5 \\
\hline Model B & 81.11 & 89.05 & Model B & 75.0 & 97.5 \\
\hline Model C & 65.95 & 84.15 & Model C & 71.0 & 104.4 \\
\hline Model D & 79.51 & 93.33 & Model D & 84.9 & 101.2 \\
\hline \multicolumn{6}{|c|}{$(\mathrm{L} / \mathrm{R}=0.3)$} \\
\hline $\begin{array}{c}\text { Ratio Of }\left(\sigma_{\mathrm{Bf}}\right) \\
\text { With(Additional } \\
\text { Mid / End) } \\
\text { Bracings }\end{array}$ & $\begin{array}{c}\text { G1 } \\
\%\end{array}$ & $\begin{array}{c}\text { G4 } \\
\%\end{array}$ & $\begin{array}{c}\text { Ratio Of ( } \sigma \\
\text { ts) } \\
\text { (Additional } \\
\text { Mid / End) } \\
\text { Bracings } \\
\end{array}$ & $\begin{array}{c}\text { G1 } \\
\%\end{array}$ & $\begin{array}{c}\text { G4 } \\
\%\end{array}$ \\
\hline I-section & 41.14 & 68.91 & I-section & 39.6 & 65.4 \\
\hline Model A & 51.34 & 81.42 & Model A & 47.4 & 79.3 \\
\hline Model B & 58.97 & 88.21 & Model B & 49.0 & 79.7 \\
\hline Model C & 61.82 & 80.05 & Model C & 56.9 & 90.9 \\
\hline Model D & 66.14 & 84.17 & Model D & 59.0 & 90.2 \\
\hline \multicolumn{6}{|c|}{$(\mathrm{L} / \mathrm{R}=\mathbf{0 . 4})$} \\
\hline $\begin{array}{c}\text { Ratio Of }\left(\sigma_{\mathrm{Bf}}\right) \\
\text { With(Additional } \\
\text { Mid / End) } \\
\text { Bracings }\end{array}$ & $\begin{array}{c}\text { G1 } \\
\%\end{array}$ & $\begin{array}{c}\text { G4 } \\
\%\end{array}$ & $\begin{array}{c}\text { Ratio Of ( } \sigma \\
\text { ts) } \\
\text { (Additional } \\
\text { Mid / End) } \\
\text { Bracings } \\
\end{array}$ & $\begin{array}{c}\text { G1 } \\
\%\end{array}$ & $\begin{array}{c}\text { G4 } \\
\%\end{array}$ \\
\hline I-section & 47.37 & 79.70 & I-section & 29.9 & 65.2 \\
\hline Model A & 49.76 & 80.15 & Model A & 35.3 & 77.1 \\
\hline Model B & 58.78 & 92.40 & Model B & 48.7 & 86.6 \\
\hline Model C & 51.54 & 72.54 & Model C & 47.8 & 97.9 \\
\hline
\end{tabular}

Table 36: Span 36m

\begin{tabular}{|c|c|c|c|c|c|}
\hline \multicolumn{6}{|c|}{$(\mathrm{L} / \mathrm{R}=\mathbf{0 . 1})$} \\
\hline $\begin{array}{c}\text { Ratio Of }\left(\sigma_{\mathrm{Bf}}\right) \\
\text { With(Additional } \\
\text { Mid / End) } \\
\text { Bracings } \\
\end{array}$ & $\begin{array}{c}\text { G1 } \\
\%\end{array}$ & $\begin{array}{c}\text { G4 } \\
\%\end{array}$ & $\begin{array}{c}\text { Ratio Of }\left(\sigma_{\mathrm{ts}}\right) \\
\text { (Additional } \\
\text { Mid / End) } \\
\text { Bracings } \\
\end{array}$ & $\begin{array}{c}\text { G1 } \\
\%\end{array}$ & $\begin{array}{c}\text { G4 } \\
\%\end{array}$ \\
\hline I-section & 67.5 & 76.8 & I-section & 85.2 & 103.8 \\
\hline Model A & 3.2 & 85.7 & $\mathrm{~A}$ & 32.7 & 06.9 \\
\hline Model B & 83.9 & 95.6 & odel B & 87.6 & 105.0 \\
\hline Model C & 76.9 & 86.8 & Model C & 86.3 & 109.7 \\
\hline Model D & 85.3 & 92.9 & Model D & 92.4 & 108.6 \\
\hline \multicolumn{6}{|c|}{$(\mathrm{L} / \mathrm{R}=\mathbf{0 . 2})$} \\
\hline $\begin{array}{c}\text { Ratio Of }\left(\sigma_{\mathrm{Bf}}\right) \\
\text { With(Additional } \\
\text { Mid / End) } \\
\text { Bracings } \\
\end{array}$ & $\begin{array}{c}\text { G1 } \\
\%\end{array}$ & $\begin{array}{c}\text { G4 } \\
\%\end{array}$ & $\begin{array}{r}\text { Ratio } \\
\text { (Ad } \\
\text { Mid } \\
\text { Br }\end{array}$ & $\begin{array}{c}\text { G1 } \\
\%\end{array}$ & $\begin{array}{c}\text { G4 } \\
\%\end{array}$ \\
\hline I-section & 72.3 & 90.4 & tion & 144.3 & 100.2 \\
\hline & 56.9 & 80.7 & & 62.2 & 97.0 \\
\hline & 69.0 & 91.5 & & 72.1 & 104.4 \\
\hline $\mathrm{C}$ & 69.3 & 82.0 & & 76.2 & 115.1 \\
\hline ID & 73.4 & 87.9 & & 106.8 & 114.2 \\
\hline \multicolumn{6}{|c|}{$(\mathrm{L} / \mathrm{R}=0.3)$} \\
\hline $\begin{array}{c}\text { Ratio Of }\left(\sigma_{\mathrm{Bf}}\right) \\
\text { With(Additional } \\
\text { Mid / End) } \\
\text { Bracings }\end{array}$ & $\begin{array}{c}\text { G1 } \\
\%\end{array}$ & $\begin{array}{c}\text { G4 } \\
\%\end{array}$ & $\begin{array}{r}\text { Ratio } \\
\text { (Ad } \\
\text { Mi } \\
\text { Br }\end{array}$ & $\begin{array}{c}\text { G1 } \\
\%\end{array}$ & $\begin{array}{c}\text { G4 } \\
\%\end{array}$ \\
\hline I-section & 52.1 & 73.6 & ion & 34.0 & 92.8 \\
\hline Model A & 34.0 & 56.1 & el A & 38.6 & 110.0 \\
\hline Model B & 54.8 & 86.0 & el B & 55.1 & 91.8 \\
\hline & 47.1 & 72.8 & & 50.0 & 114.1 \\
\hline Mod & 59.8 & 82.7 & Mo & 68.4 & 121.2 \\
\hline \multicolumn{6}{|c|}{$(\mathrm{L} / \mathrm{R}=0.4)$} \\
\hline $\begin{array}{c}\text { Ratio Of }\left(\sigma_{\mathrm{Bf}}\right) \\
\text { With(Additional } \\
\text { Mid / End) } \\
\text { Bracings } \\
\end{array}$ & $\begin{array}{c}\text { G1 } \\
\%\end{array}$ & $\begin{array}{c}\text { G4 } \\
\%\end{array}$ & $\begin{array}{c}\text { Ratio Of }\left(\sigma_{\mathrm{ts}}\right) \\
\text { (Additional } \\
\text { Mid / End) } \\
\text { Bracings }\end{array}$ & $\begin{array}{c}\text { G1 } \\
\%\end{array}$ & $\begin{array}{c}\text { G4 } \\
\%\end{array}$ \\
\hline I-section & 31.7 & 72.0 & tion & 20.8 & 67.5 \\
\hline & 34.9 & 74.0 & & 38.0 & 80.2 \\
\hline Model B & 48.1 & 84.2 & Model B & 40.6 & 91.9 \\
\hline Model C & 40.8 & 73.9 & Model C & 36.2 & 105.4 \\
\hline Model D & 56.8 & 83.7 & Model D & 71.5 & 118.4 \\
\hline
\end{tabular}

Tables (37) to (39) show the ratio of vertical displacement in the mid span of I-section and proposed models between decks modeled with different number of cross bracings.

Table (37): Span $24 \mathrm{~m}$

\begin{tabular}{|c|c|c|c|c|c|}
\hline \multicolumn{3}{|c|}{$(\mathrm{L} / \mathrm{R}=\mathbf{0 . 1})$} & \multicolumn{3}{|c|}{$(\mathrm{L} / \mathrm{R}=0.2)$} \\
\hline \begin{tabular}{|} 
Ratio Of Vertical \\
Displacement \\
With (Additional \\
Mid Bracings / \\
End Bracings) For
\end{tabular} & $\begin{array}{c}\text { G1 } \\
\%\end{array}$ & $\begin{array}{c}\text { G4 } \\
\%\end{array}$ & \begin{tabular}{|} 
Ratio Of Vertical \\
Displacement \\
With (Additional \\
Mid Bracings / \\
End Bracings) For
\end{tabular} & $\begin{array}{c}\text { G1 } \\
\%\end{array}$ & $\begin{array}{c}\text { G4 } \\
\%\end{array}$ \\
\hline I-section & 80.4 & 81.9 & I-section & 59.4 & 65.1 \\
\hline Model A & 90.9 & 96.0 & Model A & 76.5 & 86.2 \\
\hline Model B & 95.2 & 98.6 & Model B & 77.3 & 105.8 \\
\hline Model C & 95.1 & 99.1 & Model C & 84.3 & 93.3 \\
\hline Model D & 98.7 & 100.5 & Model D & 94.6 & 99.1 \\
\hline \multicolumn{3}{|c|}{$(\mathrm{L} / \mathrm{R}=\mathbf{0 . 3 )}$} & \multicolumn{3}{|c|}{$(\mathrm{L} / \mathrm{R}=0.4)$} \\
\hline \begin{tabular}{|l|} 
Ratio Of Vertical \\
Displacement \\
With (Additional \\
Mid Bracings / \\
End Bracings) For
\end{tabular} & $\begin{array}{c}\text { G1 } \\
\%\end{array}$ & $\begin{array}{c}\text { G4 } \\
\%\end{array}$ & $\begin{array}{l}\text { Ratio Of Vertical } \\
\text { Displacement } \\
\text { With (Additional } \\
\text { Mid Bracings / } \\
\text { End Bracings) For }\end{array}$ & $\begin{array}{c}\text { G1 } \\
\%\end{array}$ & $\begin{array}{c}\text { G4 } \\
\%\end{array}$ \\
\hline I-section & 47.5 & 57.2 & I-section & 40.8 & 53.6 \\
\hline Model A & 64.6 & 78.1 & Model A & 55.9 & 72.0 \\
\hline
\end{tabular}

\section{Volume 5 Issue 3, March 2016}




\section{International Journal of Science and Research (IJSR)}

ISSN (Online): 2319-7064

Index Copernicus Value (2013): 6.14 | Impact Factor (2014): 5.611

\begin{tabular}{|l|l|l|l|l|l|}
\hline Model B & 79.4 & 88.8 & Model B & 73.6 & 85.2 \\
\hline Model C & 75.1 & 87.7 & Model C & 64.5 & 80.7 \\
\hline Model D & 90.7 & 97.4 & Model D & 86.9 & 95.4 \\
\hline
\end{tabular}

Table 38: Span 30m

\begin{tabular}{|c|c|c|c|c|c|}
\hline \multicolumn{3}{|c|}{$(\mathrm{L} / \mathrm{R}=\mathbf{0 . 1})$} & \multicolumn{3}{|c|}{$(\mathrm{L} / \mathrm{R}=\mathbf{0 . 2})$} \\
\hline \begin{tabular}{|} 
Ratio Of Vertical \\
Displacement \\
With(Additional \\
Mid Bracings / \\
End Bracings) \\
For
\end{tabular} & $\begin{array}{c}\text { G1 } \\
\%\end{array}$ & $\begin{array}{c}\text { G4 } \\
\%\end{array}$ & $\begin{array}{l}\text { Ratio Of Vertical } \\
\text { Displacement } \\
\text { With(Additional } \\
\text { Mid Bracings / End } \\
\text { Bracings) For }\end{array}$ & $\begin{array}{c}\text { G1 } \\
\%\end{array}$ & $\begin{array}{c}\text { G4 } \\
\%\end{array}$ \\
\hline I-section & 70.5 & 73.2 & I-section & 46.0 & 54.7 \\
\hline Model A & 84.8 & 92.5 & Model A & 63.5 & 77.5 \\
\hline Model B & 89.9 & 96.5 & Model B & 72.9 & 83.0 \\
\hline Model C & 89.2 & 96.5 & Model C & 69.7 & 84.6 \\
\hline Model D & 95.0 & 100 & Model D & 81.8 & 94.1 \\
\hline \multicolumn{3}{|c|}{$(\mathrm{L} / \mathrm{R}=0.3)$} & \multicolumn{3}{|c|}{$(\mathrm{L} / \mathrm{R}=0.4)$} \\
\hline $\begin{array}{c}\text { Ratio Of Vertical } \\
\text { Displacement } \\
\text { With (Additional } \\
\text { Mid Bracings / } \\
\text { End Bracings) } \\
\text { For }\end{array}$ & $\begin{array}{c}\text { G1 } \\
\%\end{array}$ & $\begin{array}{c}\text { G4 } \\
\%\end{array}$ & $\begin{array}{l}\text { Ratio Of Vertical } \\
\text { Displacement With } \\
\text { (Additional Mid } \\
\text { Bracings / End } \\
\text { Bracings) For }\end{array}$ & $\begin{array}{c}\text { G1 } \\
\%\end{array}$ & $\begin{array}{c}\text { G4 } \\
\%\end{array}$ \\
\hline I-section & 31.4 & 43.8 & I-section & 27.3 & 42.0 \\
\hline Model A & 47.0 & 65.5 & Model A & 38.9 & 59.6 \\
\hline Model B & 52.6 & 76.2 & Model B & 52.3 & 72.2 \\
\hline Model C & 54.4 & 74.3 & Model C & 51.0 & 73.8 \\
\hline Model D & 53.5 & 75.3 & Model D & 69.2 & 87.8 \\
\hline
\end{tabular}

Table 39: Span 36m

\begin{tabular}{|c|c|c|c|c|c|}
\hline \multicolumn{3}{|c|}{$(\mathrm{L} / \mathrm{R}=0.1)$} & \multicolumn{3}{|c|}{$(\mathrm{L} / \mathrm{R}=0.2)$} \\
\hline $\begin{array}{c}\text { Ratio Of Vertical } \\
\text { Displacement } \\
\text { With (Additional } \\
\text { Mid Bracings / } \\
\text { End Bracings) For }\end{array}$ & $\begin{array}{c}\text { G1 } \\
\%\end{array}$ & $\begin{array}{c}\text { G4 } \\
\%\end{array}$ & $\begin{array}{c}\text { Ratio Of Vertical } \\
\text { Displacement With } \\
\text { (Additional Mid } \\
\text { Bracings / End } \\
\text { Bracings) For }\end{array}$ & $\begin{array}{c}\text { G1 } \\
\%\end{array}$ & $\begin{array}{c}\text { G4 } \\
\%\end{array}$ \\
\hline I-section & 67.8 & 71.7 & I-section & 48.2 & 59.3 \\
\hline Model A & 80.7 & 90.0 & Model A & 57.7 & 73.8 \\
\hline Model B & 86.5 & 95.2 & Model B & 68.3 & 83.9 \\
\hline Model C & 86.2 & 94.7 & Model C & 69.3 & 85.2 \\
\hline Model D & 92.9 & 99.6 & Model D & 81.9 & 94.8 \\
\hline \multicolumn{3}{|c|}{$(\mathrm{L} / \mathrm{R}=0.3)$} & \multicolumn{3}{|c|}{$(\mathrm{L} / \mathrm{R}=0.4)$} \\
\hline $\begin{array}{l}\text { Ratio Of Vertical } \\
\text { Displacement } \\
\text { With (Additional } \\
\text { Mid Bracings / } \\
\text { End Bracings) For }\end{array}$ & $\begin{array}{c}\text { G1 } \\
\%\end{array}$ & $\begin{array}{c}\text { G4 } \\
\%\end{array}$ & $\begin{array}{c}\text { Ratio Of Vertical } \\
\text { Displacement With } \\
\text { (Additional Mid } \\
\text { Bracings / End } \\
\text { Bracings) For }\end{array}$ & $\begin{array}{c}\text { G1 } \\
\%\end{array}$ & $\begin{array}{c}\text { G4 } \\
\%\end{array}$ \\
\hline I-section & 30.2 & 45.4 & I-section & 22.3 & 38.9 \\
\hline Model A & 39.9 & 60.0 & Model A & 30.6 & 53.4 \\
\hline Model B & 50.9 & 70.9 & Model B & 40.8 & 63.8 \\
\hline Model C & 48.8 & 71.1 & Model C & 41.2 & 62.4 \\
\hline Model D & 64.6 & 84.9 & Model D & 83.2 & 79.9 \\
\hline
\end{tabular}

\section{Conclusions}

1)The proposed finite element models used for predicting the behavior of the new types of steel girders system in curved simply supported composite decks using SAP2000 v.14 have proved their efficiency in analyses of such types of bridges.

2) The proposed new models of steel girders system show good strength in torsion compared to the typical I-section. The maximum gained strength (in stresses and deflections)

is with model $\mathrm{D}$ of trapezoidal bottom flange shape filled with concrete.

3)The parametric study was to verify the behavior of proposed models compared to that of I section girders with the same geometry and loading conditions and the conclusions derived from this parametric study are:

5.1 Maximum enhancement ratio of tensile stresses in bottom flange for each proposed model and typical I-section is:

- (47.7\%) for model A and typical I-section for span $24 \mathrm{~m}$ with $(\mathrm{L} / \mathrm{R}=0.3)$ and with end bracings only.

- (42.76\%) for model B and typical I-section for span $24 \mathrm{~m}$ with $(\mathrm{L} / \mathrm{R}=0.3)$ and with end bracings only.

- (36.12\%) for model C and typical I-section for span $24 \mathrm{~m}$ with $(\mathrm{L} / \mathrm{R}=0.3)$ and with end bracings only.

- (32.17\%) for model D and typical I-section for span $24 \mathrm{~m}$ with $(\mathrm{L} / \mathrm{R}=0.3)$ and with end bracings only.

5.2. Maximum enhancement ratio for vertical displacement is:

- (71.2\%) for model A and typical I-section for span 30m with $(\mathrm{L} / \mathrm{R}=0.3)$ and with end bracings only.

- (66.6\%) for model B and typical I-section for span $30 \mathrm{~m}$ with $(\mathrm{L} / \mathrm{R}=0.3)$ and with end bracings only.

- (39.1\%) for model C and typical I-section for span 30m with $(\mathrm{L} / \mathrm{R}=0.4)$ and with end bracings only.

- (29.5\%) for model D and typical I-section for span $36 \mathrm{~m}$ with $(\mathrm{L} / \mathrm{R}=0.4)$ and with end bracings only.

5.3. The maximum percentage of increase of tensile stresses of bottom flange between girders with mid bracings and those with end bracings only is in girder model A with value (34\%) for span $36 \mathrm{~m}$ with (L/R=0.3).

5.4. The maximum percentage of increase of vertical displacements between girders with mid bracings and those with end bracings only is in girder model A with value (30.6\%) for span $36 \mathrm{~m}$ with $(\mathrm{L} / \mathrm{R}=0.4)$.

\section{References}

[1] American Association for State Highway and Transportation Officials, AASHTO. AASHTO ASD Bridge Design Specifications , 17th Edition, 2001. Washington, D.C.

[2] American Association for State Highway and Transportation Officials, AASHTO. 2004. AASHTO LRFD Bridge Design Specifications. Washington, D.C.

[3] Dong J., Sause R., "Finite Element Analysis of Curved Tubular Flange Girders." Engineering Structures 32(1): 319-327, (2010a).

[4] Putnam E., "Design, Experimental, and Analytical Study of a Horizontally Curved Tubular Flange Girder." M.Sc. Thesis, Department of Civil and Environmental Engineering, Lehigh University, Bethlehem, PA, (2010).

[5] Asal Mohammed , " Behavior of Hollow Tubular Flange Girder System in Steel Curved Bridges" M.Sc. Thesis , Civil Engineering Department, Al Nahrain University, (2014). 